\title{
Analysis of Moving Object Imaging from Compressively Sensed SAR Data in the Presence of Dictionary Mismatch
}

\author{
Ahmed Shaharyar Khwaja, ${ }^{1}$ Muhammad Naeem, ${ }^{1,2}$ and Alagan Anpalagan ${ }^{1}$ \\ ${ }^{1}$ Department of Electrical and Computer Engineering, Ryerson University, Toronto, ON, Canada M5B 2 K3 \\ ${ }^{2}$ Department of Electrical Engineering, COMSATS Institute of IT, Wah Campus, Wah 47040, Pakistan \\ Correspondence should be addressed to Ahmed Shaharyar Khwaja; akhwaja@ee.ryerson.ca
}

Received 4 April 2013; Revised 4 October 2013; Accepted 6 October 2013

Academic Editor: Krzysztof Kulpa

Copyright (c) 2013 Ahmed Shaharyar Khwaja et al. This is an open access article distributed under the Creative Commons Attribution License, which permits unrestricted use, distribution, and reproduction in any medium, provided the original work is properly cited.

\begin{abstract}
We present compressed sensing (CS) synthetic aperture radar (SAR) moving target imaging in the presence of dictionary mismatch. Unlike existing work on CS SAR moving target imaging, we analyze the sensitivity of the imaging process to the mismatch and present an iterative scheme to cope with dictionary mismatch. We analyze and investigate the effects of mismatch in range and azimuth positions, as well as range velocity. The analysis reveals that the reconstruction error increases with the mismatch and range velocity mismatch is the major cause of error. Instead of using traditional Laplacian prior (LP), we use Gaussian-Bernoulli prior (GBP) for CS SAR imaging mismatch. The results show that the performance of GBP is much better than LP. We also provide the Cramer-Rao Bounds (CRB) that demonstrate theoretically the lowering of mean square error between actual and reconstructed result by using the GBP. We show that a combination of an upsampled dictionary and the GBP for reconstruction can deal with position mismatch effectively. We further present an iterative scheme to deal with the range velocity mismatch. Numerical and simulation examples demonstrate the accuracy of the analysis as well as the effectiveness of the proposed upsampling and iterative scheme.
\end{abstract}

\section{Introduction}

According to compressed sensing (CS) [1-3] theory, randomly undersampled signals can be reconstructed using linear programming [1], orthogonal matching pursuit (OMP) [4], and Bayesian methods [5-7]. The advantages gained by using CS are hardware simplification [8], reduction in equipment cost, data size, and acquisition time $[9,10]$, and deblurring and enhancing resolution from incomplete measurements [11].

Compressed sensing for synthetic aperture radar (SAR) is an active area of research for remote sensing. The use of CS based reconstruction can have an impact on the design of high resolution SAR systems as these systems encounter hardware design problems and require significant processing [12]. CS has been applied for imaging of static objects in through-the-wall SAR imaging [13-15], tomographic SAR imaging [16-18], and SAR image formation with reduced data [19], where advantage is taken of the fact that the observed scenes are sparse. The static scenes may not always be sparse. The scenes containing a few strong intensity moving scatterers in a weak stationary background present an opportunity for CS application as they are inherently sparse. These moving targets suffer from position displacement and defocusing due to motion [20]. The use of CS can help in reducing acquired data size as well as simultaneous motion parameter estimation imaging with reduced data. Sparsity can be further enhanced using clutter cancelation where the static parts of an observed scene are suppressed [21].

Compressed sensing for SAR moving object imaging has become an active area of research. References [22, 23] apply CS for moving target parameter estimation by defining a dictionary based on the response of moving objects for different motion parameters. Both of these references use clutter cancelation to enhance sparsity. Reference [24] makes use of distributed CS applied to along-track interferometric SAR data for moving target imaging and shows that distributed CS can offer better performance with less samples compared 
TABLE 1: Comparison of existing references.

\begin{tabular}{|c|c|c|c|c|}
\hline References & SAR moving target & Prior & Dictionary mismatch & Remarks \\
\hline$[6]$ & No & GBP & Yes & $\begin{array}{l}\text { Superior performance of GBP over LP is shown by } \\
\text { simulations }\end{array}$ \\
\hline$[13,14]$ & No & LP & No & Apply CS for through-the-wall imaging \\
\hline [15] & No & LP & Yes & $\begin{array}{l}\text { Apply CS for through-the-wall imaging. } \\
\text { Performance degradation due to pixel mismatch and } \\
\text { wave propagation velocity shown by simulations }\end{array}$ \\
\hline [16-18] & No & LP & No & Apply CS for tomographic SAR imaging \\
\hline [19] & No & LP & No & Apply CS for focusing of static scenes \\
\hline [7] & No & GBP & Yes & $\begin{array}{l}\text { Fast implementation of GBP reconstruction shows } \\
\text { superior performance by simulations }\end{array}$ \\
\hline$[21]$ & No & LP & No & $\begin{array}{l}\text { Suggest clutter cancelation to enhance sparsity of a } \\
\text { scene containing moving targets }\end{array}$ \\
\hline$[22]$ & Yes & LP & No & Apply CS for motion estimation \\
\hline$[23]$ & Yes & LP & Yes & $\begin{array}{l}\text { Apply CS for motion estimation, performance } \\
\text { degradation due to range velocity mismatch shown } \\
\text { by simulations }\end{array}$ \\
\hline [24] & Yes & LP & No & Apply distributed CS for motion estimation \\
\hline$[25]$ & Yes & LP & Yes & $\begin{array}{l}\text { Apply CS for motion estimation; simulations show } \\
\text { no performance degradation due to velocity } \\
\text { mismatch }\end{array}$ \\
\hline$[26,27]$ & No & LP & Yes & $\begin{array}{l}\text { Performance degradation due to dictionary } \\
\text { mismatch shown by simulations and theory }\end{array}$ \\
\hline
\end{tabular}

to traditional CS. Reference [25] uses CS for moving target parameter estimation for mono- and multistatic SAR configurations and simulated data. These references show that CS can achieve imaging of moving objects as well as moving object parameter estimation when SAR data are sampled at a rate less than the traditional Nyquist sampling rate.

Compressed sensing reconstruction algorithms use a dictionary in which the reconstructed signal is assumed to be sparse. However, the dictionary in which the signal is actually sparse may be different and the resulting dictionary mismatch causes a performance degradation $[26,27]$. In order to apply CS for practical applications, it is necessary to study the reconstruction performance degradation in the presence of dictionary mismatch. Reference [26] shows that dictionary mismatch can be seen equivalent to multiplicative noise. It also shows that reconstruction error increases linearly with mismatch. Reference [27] considers the effect of dictionary mismatch in CS reconstruction. It shows that, in case of using a Fourier dictionary, reconstruction performance degrades considerably when a mismatch exists. Due to this reason, it recommends examining the effects of mismatch on radar imaging. Reference [15] has shown performance degradation by means of imaging examples for static targets in the presence of mismatch in position and wave propagation velocity. The authors in [15] also state that they are extending the initial results presented in [28] for dealing with position mismatch in through-the-wall imaging.

According to the best of our knowledge, dictionary mismatch analysis has not been done theoretically for CS moving target SAR imaging in the presence of position and range velocity mismatch. A summary of the main features of the existing references is given in Table 1 . It shows that, in the existing literature, the theoretical analysis of the effects of dictionary mismatch for moving target CS SAR imaging have not been carried out. Therefore, it remains an open problem. It further shows that a prior other than Laplacian prior (LP), for example, Gaussian-Bernoulli prior (GBP), for CS moving target imaging has not been used. Similarly, a theoretical analysis to show the advantage of the prior in dealing with dictionary mismatch is also missing. In [29], we have partially studied this problem and its effects for SAR and inverse SAR. We showed that dictionary generation using upsampled parameters is required to deal with errors arising due to mismatch in positions and range velocity.

The emphasis of this paper is to show the performance degradation in case of a target moving in the range direction. The dictionary mismatch arising due to discretization and dictionary size considerations causes performance degradation in terms of mean square error (MSE) between actual and reconstructed results, especially when there is a range velocity mismatch. We examine reasons for this degradation and also show theoretically and experimentally that using GBP for CS reconstruction compared to the traditionally used LP can compensate for some amount of mismatch. The motivation of using a different prior is to make use of extra information in improving reconstructed image quality as shown in [30]. We propose to deal with CS SAR moving target imaging in the presence of dictionary mismatch due to positions and range velocity. The main contributions of this paper are as follows.

(1) We analyze dictionary mismatch and its effects theoretically, show MSE calculated from simulated SAR 
data for different types of mismatch in range and azimuth pixels as well as range velocity, and give parameter resolution limits for maintaining a reasonable level of reconstruction accuracy. We show that CS SAR moving target imaging is very sensitive to range velocity mismatch.

(2) We analyze the problem by means of Cramer-Rao Bounds (CRB) and show theoretically that reconstruction with Gaussian-Bernoulli prior (CSGBP) instead of traditional Laplacian prior (CSLP) can deal with some mismatch effectively.

(3) We present simulation results using CSGBP reconstruction and show that its use can lead to lower MSE, especially when the dictionary mismatch is small. This can be used to deal with position mismatch and reduce upsampling in positions that is required to counter mismatch effects.

(4) We also propose to reconstruct in the presence of range-velocity mismatch using an iterative scheme, where dictionaries with different range velocities are created efficiently. The contrast of the reconstructed result is maximized.

We would also like to point out that we deal specifically with the case of pulsed SAR. Any extension of dictionary mismatch effects and parameter resolution calculations to other types of SAR will need to take into account the difference in imaging mechanism; for example, in case of continuous wave SAR, it is known that range velocity creates a shift in the range direction, which is absent in pulsed SAR. Therefore, results for mismatch analysis and resolutions in range position and range velocity will need to take this additional shift into account.

This paper is organized as follows. Section 2 presents the data model and formulation of moving target velocity estimation problem in case of CS SAR. Section 3 analyzes the effects of different kinds of dictionary mismatch, that is, range and azimuth positions and range velocity on CS SAR moving target imaging. Section 4 presents numerical and imaging examples to present the effects of dictionary mismatch in terms of MSE as well as the accuracy of the analysis and the effectiveness of the proposed method. Conclusions are given in Section 5.

\section{System Model and Problem Formulation}

In this paper, $x$ denotes a scalar, $\mathbf{x}$ denotes a vector, and $\mathbf{X}$ denotes a matrix. We use $\mathbf{X}^{H}$ and $\mathbf{X}^{T}$ to denote conjugate transpose and transpose of $\mathbf{X}$, respectively. The same notation is used for Greek characters; that is, $\sigma$ denotes a scalar, $\boldsymbol{\sigma}$ denotes a vector, and $\Sigma$ denotes a matrix. We use $\Sigma^{H}$ and $\Sigma^{T}$ to denote conjugate transpose and transpose of $\boldsymbol{\Sigma}$, respectively. The function $\operatorname{diag}(\mathbf{x})$ represents a function that converts a vector $\mathbf{x}$ of size $N_{t} \times 1$ into a diagonal matrix of size $N_{t} \times N_{t}$ and $\operatorname{det}(\mathbf{X})$ represents the determinant of the matrix $\mathbf{X}$.
Synthetic aperture radar consists of an antenna mounted on a moving platform [31]. A pulsed SAR sends electromagnetic pulse $p(t)$ at a carrier frequency $f_{c}$ and a chirp rate $K$. The pulse length is denoted by $T_{p}$. This pulse is given as

$$
p(t)=\operatorname{rect}\left(\frac{t}{T_{p}}\right) \exp \left(j 2 \pi f_{c} t-j \pi K t^{2}\right),
$$

where

$$
\begin{aligned}
\operatorname{rect}\left(\frac{t}{T_{p}}\right) & =1, \quad \text { if } 0 \leq t \leq T_{p} \\
& =0, \quad \text { otherwise, }
\end{aligned}
$$

and $t=t_{1}, t_{2}, t_{3}, \ldots, t_{N_{r}}$. The signals are reflected from each scatterer in the observed scene. Let $\sigma_{0}$ be a sparse vector of size $N_{t} \times 1$ that contains reflectivities for each point in the scene having different motion parameters. $\Psi_{\mathbf{0}}$ is an $N_{s} \times N_{t}$ matrix in which the signal is actually sparse and contains response of moving targets for every point in the scene with each considered motion parameter. Let $\Phi$ be a sampling matrix of size $M \times N_{s}$, where $M<N_{s}$. This represents the case where the number of measurements is less than the required sampling rate due to data loss or intentionally reduced data acquisition to simplify the acquisition hardware [10], such as analog-to-digital converter. With different sampling configurations, one can get reasonable image reconstruction [13]. In this paper, we use undersampling in range direction as measurement operator. The raw data signal model can be written in one-dimensional form as [29]

$$
\mathrm{s}_{0}=\Phi \Psi_{0} \sigma_{0}+\varepsilon
$$

where $\boldsymbol{\varepsilon}$ denotes measurement noise. $\boldsymbol{\Psi}_{\mathbf{0}}$ contains the response of each moving point in $1 \mathrm{D}$ form. This response for $n$th moving point having $k$ th range velocity is given as [29]

$$
\begin{aligned}
\mathbf{s}_{n}^{k}= & {\left[\widehat{s}_{n}^{k}\left(t_{1}, \tau_{1}\right), \vec{s}_{n}^{k}\left(t_{2}, \tau_{1}\right), \ldots, \widehat{s}_{n}^{k}\left(t_{N_{r}}, \tau_{1}\right),\right.} \\
& \widehat{s}_{n}^{k}\left(t_{1}, \tau_{2}\right), \widetilde{s}_{n}^{k}\left(t_{2}, \tau_{2}\right), \ldots, \widehat{s}_{n}^{k}\left(t_{N_{r}}, \tau_{2}\right), \ldots, \\
& \left.\widehat{s}_{n}^{k}\left(t_{1}, \tau_{N_{y}}\right), \vec{s}_{n}^{k}\left(t_{2}, \tau_{N_{y}}\right), \ldots, \widetilde{s}_{n}^{k}\left(t_{N_{r}}, \tau_{N_{y}}\right)\right]^{T},
\end{aligned}
$$

where

$$
\begin{aligned}
\tilde{s}_{n}^{k}\left(t_{l}, \tau_{m}\right)= & p\left(t_{l}-\frac{2 d_{n}^{k}\left(\tau_{m}\right)}{c}\right) \\
= & \operatorname{rect}\left(\frac{t_{l}-2 d_{n}^{k}\left(\tau_{m}\right) / c}{T_{p}}\right) \exp \left\{-j k_{c} d_{n}^{k}\left(\tau_{m}\right)\right\} \\
& \times \exp \left\{-j \pi K\left(t_{l}-\frac{2 d_{n}^{k}\left(\tau_{m}\right)}{c}\right)^{2}\right\} \\
d_{n}^{k}\left(\tau_{m}\right)= & \sqrt{\left(x_{n}-v_{x}^{k} \tau_{m}\right)^{2}+h^{2}+\left(y_{n}-V \tau_{m}\right)^{2}} .
\end{aligned}
$$


The size of $\mathbf{s}_{n}^{k}$ is $N_{s} \times 1 . \Psi_{\mathbf{0}}$ is generated for initial velocity $v_{x i}$ and final velocity $v_{x f}$ for a total number of $N v_{x}$ range velocities. The dictionary element corresponding to a velocity $v_{x}^{1}$ is as follows:

$$
\Psi_{0}^{1}=\left[\mathbf{s}_{1}^{1} \cdots \mathbf{s}_{N_{s}}^{1}\right]
$$

The final dictionary $\Psi_{\mathbf{0}}$ is stored in an $N_{s} \times N_{t}$ matrix given as

$$
\Psi_{0}=\left[\Psi_{0}^{1}|\cdots| \Psi_{0}^{N v_{x}}\right]
$$

Due to undersampling, the problem of recovering $\sigma_{0}$ from $\mathbf{s}_{\mathbf{0}}$ becomes an underdetermined problem. We can solve this problem by including a-priori information for getting the solution; for example, select a solution such that the number of nonzero coefficients is the smallest. This can be expressed as follows:

$$
\min \left\|\sigma_{0}\right\|_{0} \quad \text { subject to } s_{0}=\Phi \Psi_{0} \sigma_{0} \text {. }
$$

The number of nonzero coefficients is denoted by $\left\|\sigma_{0}\right\|_{0}$, known as $l_{0}$ norm. However, this minimization problem is nonconvex, which means that finding a global solution is difficult or not guaranteed. In addition, it is computationally difficult to solve as it requires search over all possible combinations of the columns of $\boldsymbol{\Phi} \Psi_{\mathbf{0}}$. To deal with these issues, we use $l_{1}$ norm minimization. This minimization is a convex approximation of the $l_{0}$ norm minimization if a property known as restricted isometric property (RIP) is satisfied. This property essentially means that the columns formed by the matrix $\boldsymbol{\Phi} \Psi_{\mathbf{0}}$ are sufficiently decorrelated with one another. The problem can be expressed as

$$
\min \left\|\sigma_{0}\right\|_{1} \text { subject to } \mathbf{s}_{\mathbf{0}}=\Phi \Psi_{0} \sigma_{0} .
$$

In order to obtain a solution based on $l_{1}$ norm minimization, we use Laplacian prior (LP) [32] as follows:

$$
\sigma_{0} \sim \exp \left(-\left\|\sigma_{0}\right\|_{1}\right) \text {. }
$$

If noise is Gaussian with variance $s_{\varepsilon}^{2}$, the solution is obtained by

$$
\begin{aligned}
\widehat{\boldsymbol{\sigma}}_{\mathbf{0}} & =\underset{\boldsymbol{\sigma}_{0}}{\operatorname{argmax}} p\left(\boldsymbol{\sigma}_{\mathbf{0}} \mid \mathbf{s}_{\mathbf{0}}\right) \\
& =\underset{\boldsymbol{\sigma}_{0}}{\operatorname{argmax}} p\left(\mathbf{s}_{\mathbf{0}} \mid \boldsymbol{\sigma}_{\mathbf{0}}\right) p\left(\boldsymbol{\sigma}_{\mathbf{0}}\right),
\end{aligned}
$$

where

$$
p\left(\mathbf{s}_{\mathbf{0}} \mid \boldsymbol{\sigma}_{\mathbf{0}}\right)=\frac{1}{2 s_{\varepsilon^{2}}} \exp \left\{-\left\|\mathbf{s}_{\mathbf{0}}-\Phi \Psi_{0} \boldsymbol{\sigma}_{0}\right\|_{2}^{2}\right\} .
$$

The solution can be written as

$$
\widehat{\boldsymbol{\sigma}}_{\mathbf{0}}=\underset{\boldsymbol{\sigma}_{0}}{\operatorname{argmin}}\left\{-\log p\left(\mathbf{s}_{\mathbf{0}} \mid \boldsymbol{\sigma}_{\mathbf{0}}\right)-\log p\left(\boldsymbol{\sigma}_{\mathbf{0}}\right)\right\}
$$

that leads to

$$
\widehat{\boldsymbol{\sigma}}_{\mathbf{0}}=\underset{\sigma_{0}}{\operatorname{argmin}}\left\|\mathbf{s}_{\mathbf{0}}-\Phi \Psi_{\mathbf{0}} \boldsymbol{\sigma}_{\mathbf{0}}\right\|_{2}^{2}+\lambda\left\|\sigma_{0}\right\|_{1}
$$

Thus, by using LP, we include the $l_{1}$ norm minimization in the solution. The parameter $\lambda$ gives weight to a priori sparse information. Equation (15) can be solved using different recovery methods, for example, linear programming and OMP. The reconstructed result $\widehat{\boldsymbol{\sigma}}_{\mathbf{0}}$ is of size $N_{t} \times 1$ and can be written as

$$
\widehat{\boldsymbol{\sigma}}_{\mathbf{0}}=\left[\widehat{\sigma}_{0,1}^{1} \cdots \widehat{\sigma}_{0, N}^{1} \cdots \widehat{\sigma}_{0,1}^{N v_{x}} \cdots \widehat{\sigma}_{0, N}^{N v_{x}}\right]^{T}
$$

where each entry of $\widehat{\boldsymbol{\sigma}}_{\mathbf{0}}$ shows the reconstructed reflectivity for each point in the scene for one velocity value; for example, $\widehat{\sigma}_{0,1}^{1}$ represents the reflectivity for a point at position $\left(r_{1}, y_{1}\right)$ and having a velocity $v_{x}^{1}$. The result can be rearranged into $N v_{x}$ 2D matrices, each having a size $n_{r} \times n_{y}$, to show the estimated reflectivities at different velocities for SAR. The matrices of size $n_{r} \times n_{y}$ may also be summed to give a final focussed reconstructed result $\widehat{\Sigma}_{0}$, shown as follows:

$$
\begin{gathered}
\widehat{\boldsymbol{\Sigma}}_{\mathbf{0}}=\sum_{i=0}^{N v_{x}-N} f\left(\hat{\boldsymbol{\sigma}}_{\mathbf{0}}(i \times N+1), \widehat{\boldsymbol{\sigma}}_{\mathbf{0}}(i \times N+2), \ldots,\right. \\
\left.\widehat{\boldsymbol{\sigma}}_{\mathbf{0}}((i+1) \times N), n_{r}, n_{y}\right) .
\end{gathered}
$$

$f\left(\widehat{\boldsymbol{\sigma}}_{\mathbf{0}}, n_{1}, n_{2}\right)$ is a function that rearranges an input $\widehat{\boldsymbol{\sigma}}_{\mathbf{0}}$ into a matrix of size $n_{1} \times n_{2}$.

Dictionary mismatch can occur in the reconstruction process due to discretization of positions as well as range velocity; for example, instead of actual position of the scatterer $\left(r_{n}, y_{n}\right)$ and velocity $v_{x}^{k}$, the basis has elements corresponding to $\left(r_{n}+\Delta r_{n}, y_{n}+\Delta y_{n}\right)$ and $v_{x}^{k}+\Delta v_{x}^{k}$. Considering $\Psi$ as the mismatched dictionary, (3) can be rewritten as

$$
\mathbf{s}=\Phi \Psi \sigma+\varepsilon,
$$

and reconstruction using the mismatched dictionary $\Psi$ causes the results to be decorrelated from $\widehat{\sigma}_{0}$ shown as follows:

$$
\widehat{\boldsymbol{\sigma}}=\Psi^{H} \Psi_{0} \widehat{\boldsymbol{\sigma}}_{\mathbf{0}} .
$$

Therefore, the effects of dictionary mismatch are related to the correlation between the mismatched and the original dictionary. In the next section, we examine the effects of this correlation on the reconstruction. Furthermore, we present solutions for the recovery of $\sigma_{0}$ that can be written as

$$
\widehat{\boldsymbol{\sigma}}_{\mathbf{0}}=\underset{\boldsymbol{\sigma}_{0}, \Psi_{\mathbf{0}}}{\operatorname{argmin}}\left\|\mathbf{s}-\Phi \Psi_{\mathbf{0}} \boldsymbol{\sigma}_{\mathbf{0}}\right\|_{2}^{2}+\lambda\left\|\sigma_{\mathbf{0}}\right\|_{1},
$$

where $\Psi_{\mathbf{0}}$ is the actual dictionary. We present solutions for calculation of $\widehat{\sigma}_{0}$ using GBP that can reduce position mismatch effects, and propose an iterative scheme to recover $\widehat{\sigma}_{0}$ in the presence of range velocity mismatch.

\section{Analysis of CS Moving Target Imaging in the Presence of Dictionary Mismatch}

3.1. Effects of Position Mismatch. We consider a chirp signal that is commonly used in imaging radars and show the effects 
of position mismatch on reconstruction. The reconstruction in the presence of mismatch depends upon the correlation between the original and the mismatched dictionaries, as given by (19). Therefore, any form of mismatch will cause erroneous results due to a correlation loss. This can be seen by taking the inner product of two chirp signals $s_{1}(t)=$ $\exp \left(-j \pi K\left(t-t_{1}\right)^{2}\right)$ and $s_{2}(t)=\exp \left(-j \pi K\left(t-t_{1}\right)^{2}\right)$ having frequencies ranging from $-B / 2$ to $B / 2$. The signals are displaced with respect to each other by a duration $\Delta t_{d}=$ $t_{1}-t_{2}$. They consist of $N$ samples with sampling time $\Delta t_{s}$. The correlation $\left|\left\langle s_{2}^{H}(t), s_{1}(t)\right\rangle\right|$ is

$$
\begin{aligned}
& \left|\left\langle\exp \left(j \pi K\left(t-t_{2}\right)^{2}\right), \exp \left(-j \pi K\left(t-t_{1}\right)^{2}\right)\right\rangle\right| \\
& \quad=\left|\sum_{n=0}^{\widetilde{N}-1} \exp \left(-j 2 \pi K \Delta t_{d} n \Delta t_{s}\right)\right|,
\end{aligned}
$$

where $t \in\left\{0, \Delta t_{s}, 2 \Delta t_{s}, \ldots,(\widetilde{N}-1) \Delta t_{s}\right\} . \Delta t_{s}$ should be less than $1 / B$. Observing that $\left|\sum_{n=0}^{\widetilde{N}-1} \exp (-j x n)\right|=|\widetilde{N} \operatorname{sinc}((1 / 2) \widetilde{N} x)|$, we can write $(21)$ as

$$
\begin{aligned}
& \left|\left\langle\exp \left(-j \pi K\left(t-t_{1}\right)^{2}\right), \exp \left(j \pi K\left(t-t_{2}\right)^{2}\right)\right\rangle\right| \\
& =\widetilde{N}\left|\operatorname{sinc}\left(\pi B \Delta t_{d}\right)\right|,
\end{aligned}
$$

where $B=K \widetilde{N} \Delta t_{s}$. As the position mismatch increases, $\Delta t_{d}$ increases and, with the increase of $\Delta t_{d}$, correlation decreases. Consequently, the amplitude of the reconstructed result will be reduced by a factor of $\left|\operatorname{sinc}\left(\pi B \Delta t_{d}\right)\right|$. The result of the correlation would be 0 when

$$
\widetilde{\Delta t}_{d}=\Delta t_{s}
$$

or

$$
\widetilde{\Delta t}_{d}=\frac{1}{B}
$$

Consequently, if the two chirp signals are displaced by $\widetilde{\Delta t}_{d}$ with respect to each other, CS imaging will fail to reconstruct the correct position. An arbitrary element will be selected and cause a failure of the CS reconstruction model. Therefore, the smaller the distance between the dictionary elements, the smaller the mismatch and the better the reconstruction at the expense of larger dictionary size and higher number of computations. In effect, by making an oversampled dictionary, we can improve the reconstruction and this oversampling should be more than twice the sampling frequencies to reduce mismatch errors; that is,

$$
\Delta t_{d}^{\max }<\frac{\widetilde{\Delta t}_{d}}{2}
$$

The implication of the above result will be studied in the next section. A moving target and the effects of mismatch in range and azimuth positions as well as range velocity on the moving target reconstruction are considered.
TABLE 2: Effect of mismatch on range and azimuth positions.

\begin{tabular}{lcc}
\hline Mismatch type & Effect in range & Effect in azimuth \\
\hline Range & $\Delta r_{n}$ & $-\Delta r_{n}\left(\Delta v_{x}^{k} / V\right) \sin \theta_{n}$ \\
Azimuth & $\Delta y_{n}\left(\Delta v_{x}^{k} / V\right) \sin \theta_{n}$ & $-\Delta y_{n}$ \\
Velocity & $y_{n}\left(\Delta v_{x}^{k} / V\right) \sin \theta_{n}$ & $-r_{n}\left(\Delta v_{x}^{k} / V\right) \sin \theta_{n}$ \\
\hline
\end{tabular}

3.2. Effects of Range Position, Azimuth Position, and Range Velocity Mismatch on Reconstruction of a Moving Target. First, we consider the equivalent static position of a moving point. A moving point at an initial position of $r_{n}, y_{n}$ and having a velocity of $v_{x}^{k}$ can be equivalently seen as a static point with coordinates $r_{m}$ and $y_{m}$ and rotated with an angle $\Theta_{n}^{k}[33]$; that is:

$$
\begin{gathered}
r_{m}=r_{n}+y_{n} \Theta, \\
y_{m}=-r_{n} \Theta+y_{n},
\end{gathered}
$$

where

$$
\begin{aligned}
\Theta_{n}^{k} & =\tan ^{-1} \frac{v_{x}^{k} \sin \theta_{n}}{V} \\
& \approx \frac{v_{x}^{k} \sin \theta_{n}}{V} .
\end{aligned}
$$

Assuming that our dictionary is created with resolutions of $s_{r}, s_{y}$, and $1 \mathrm{~m} / \mathrm{s}$ in range position, azimuth position, and range velocity, respectively, the mismatch effects on a moving target can be divided into 3 categories as follows.

(i) A subpixel mismatch in range position represented as $\Delta r_{n}$. This mismatch will lead to an equivalent shift of $\Delta r_{n}$ in the range position and an equivalent shift of $-\Delta r_{n}\left(v_{x}^{k} \sin \theta_{n} / V\right)$ in the azimuth position.

(ii) A subpixel mismatch in azimuth position represented as $\Delta y_{n}$. This mismatch will lead to an equivalent shift of $\Delta y_{n}\left(v_{x n} \sin \theta_{n} / V\right)$ in the range position and an equivalent shift of $-\Delta y_{n}$ in the azimuth position.

(iii) A fraction of $\mathrm{m} / \mathrm{s}$ mismatch in range velocity represented as $\Delta v_{x}^{k}$. This mismatch will lead to an equivalent shift of $y_{n}\left(\Delta v_{x}^{k} / V\right) \sin \theta_{n}$ in the range position and an equivalent shift of $-r_{n}\left(\Delta v_{x}^{k} / V\right) \sin \theta_{n}$ in the azimuth position. As an example, if a point in acquired raw data is at position $\left(r_{n}, y_{n}\right)$ moving with a velocity $v_{x}^{k}+\Delta v_{x}^{k}$, and the dictionary contains elements with velocity $v_{x}^{k}$, the reconstructed estimate will be a point at position $\left(r_{n}+y_{n}\left(\Delta v_{x}^{k} / V\right) \sin \theta_{n}, y_{n}-\right.$ $\left.r_{n}\left(\Delta v_{x}^{k} / V\right) \sin \theta_{n}\right)$ instead of the true position of $\left(r_{n}, y_{n}\right)$. As $r_{n}$ is large, the effect on azimuth position will be more evident even when range velocity mismatch is small. The mismatch effects due to $\Delta r_{n}, \Delta y_{n}$, and $\Delta v_{x n}^{k}$ are summarized in Table 2.

3.3. Effects on Reconstruction for a Single Point in the Presence of Range Position, Azimuth Position, and Range Velocity Mismatch. Based on the above discussion, the effects of 
mismatch on reflectivity reconstruction for a single element $\boldsymbol{\sigma}_{0}=\sigma_{1}^{1} \delta\left(r-r_{1}, y-y_{1}\right)$, where $r_{1}$ and $y_{1}$ are the pixel positions, can be summarized as follows.

(i) A mismatch of $\Delta r_{1}$ will cause a shift of $\Delta r_{1}$ in range position in the reconstructed result. The shift in azimuth position $-\Delta r_{1}\left(\Delta v_{x}^{1} / V\right) \sin \theta_{1}$ can be neglected as it is small due to the presence of $V$ in the denominator. The result will be

$$
\widehat{\boldsymbol{\sigma}}_{0}=\sigma_{1}^{1} \operatorname{sinc}\left(\pi \frac{\Delta r_{1}}{s_{r}}\right) \delta\left(r-r_{1}, y-y_{1}\right)
$$

leading to a loss of amplitude.

(ii) A mismatch of $\Delta y_{1}$ will cause a shift of $\Delta y_{1}$ in azimuth position in the reconstructed result. The shift in azimuth position $\Delta y_{1}\left(\Delta v_{x}^{1} / V\right) \sin \theta_{1}$ can be neglected due to the presence of $V$ in the denominator. The result will be

$$
\widehat{\boldsymbol{\sigma}}_{0}=\sigma_{1}^{1} \operatorname{sinc}\left(\pi \frac{\Delta y_{1}}{s_{y}}\right) \delta\left(r-r_{1}, y-y_{1}\right)
$$

leading to a loss of amplitude.

(iii) A range velocity mismatch $\Delta v_{x}^{1}$ causes a large shift in azimuth from the true position, given as $\Delta y_{1}^{1}=$ $-r_{1} \Delta v_{x}^{1} \sin \theta_{1} / V$. The shift in the range position $y_{1}\left(\Delta v_{x}^{1} / V\right) \sin \theta_{1}$ can be neglected. However, the shift in azimuth position cannot be neglected due to the presence of $r_{1}$ in the numerator that is of the order of $10^{3}$ or higher. It can be further divided into 2 parts as follows.

(1) An interpixel displacement: $\left\lfloor\Delta y_{1}^{1}, s_{y}\right\rfloor$, where $\lfloor\cdot\rfloor$ is the floor operation.

(2) An intrapixel displacement: $\Delta y_{1}^{1} \bmod s_{y}$, where mod is the modulo operation.

The reconstructed result will be

$$
\begin{aligned}
\widehat{\sigma}_{0}= & \sigma_{1}^{1} \operatorname{sinc}\left(\pi \frac{\Delta y_{n}^{1} \bmod s_{y}}{s_{y}}\right) \\
& \times \delta\left(r-r_{1}, y-y_{1}+\left\lfloor\Delta y_{1}^{1}, s_{y}\right\rfloor\right)
\end{aligned}
$$

leading to a loss of amplitude and azimuth position shift.

(iv) In order to avoid the loss in amplitude as well as azimuth mispositioning of the reconstructed result, the dictionary can be created with higher parameter resolution. The dictionary resolutions in range and azimuth positions and range velocity are such that any mismatch does not lead to a misselection of elements. This can be achieved if the dictionary resolutions are less than half the pixel sizes. This ensures that a correct pixel positions is selected. These criteria can be expressed as follows for range and azimuth positions:

$$
\begin{aligned}
& \widetilde{\Delta r}<\frac{s_{r}}{2}, \\
& \widetilde{\Delta y}<\frac{s_{y}}{2} .
\end{aligned}
$$

In case of velocity, the shift in azimuth position caused by range velocity mismatch should be less than half the pixel size; that is,

$$
\Delta y_{n}^{k}<\frac{s_{y}}{2}
$$

or

$$
-r_{n} \frac{\Delta v_{x}^{k}}{V} \sin \left(\theta_{n}\right)<\frac{s_{y}}{2} .
$$

As this shift is large for a larger value of $r_{n}$, we choose the farthest slant-range distance $r_{\max }$ to get a conservative estimate as follows:

$$
\frac{r_{\max } \widetilde{\Delta v_{x}} \sin \left(\theta_{\max }\right)}{V}<\frac{s_{y}}{2},
$$

where the angle corresponding to $r_{\max }$ is $\theta_{\max }$. This leads to

$$
\widetilde{\Delta v_{x}}<\frac{s_{y} V}{r_{\max } \sin \left(\theta_{\max }\right)} .
$$

The limit given by (32) is also applicable for compensating intrapixel displacements due to velocity mismatch. Please note that due to the presence of $r_{\max }$ in the denominator, $\widetilde{\Delta v_{x}}$ is very small, which means that the dictionary needs to be created with very closely spaced velocity values.

When there is a moving scene consisting of a number of points given as

$$
\boldsymbol{\sigma}_{0}=\sum_{k} \sum_{n} \sigma_{n}^{k} \delta\left(r-r_{n}, y-y_{n}\right)
$$

the reconstructed result in the presence of mismatch is as follows:

$$
\begin{aligned}
\hat{\boldsymbol{\sigma}}_{0}= & \sum_{k} \sum_{n} \sigma_{n}^{k} \operatorname{sinc}\left(\pi \frac{\Delta r_{n}}{s_{r}}\right) \operatorname{sinc}\left(\pi \frac{\Delta y_{n}}{s_{y}}\right) \\
& \times \operatorname{sinc}\left(\pi \frac{\Delta y_{n}^{k} \bmod s_{y}}{s_{y}}\right) \delta\left(r-r_{n}, y-y_{n}+\left\lfloor\Delta y_{n}^{k}, s_{y}\right\rfloor\right) .
\end{aligned}
$$

The three sinc functions represent a loss in estimated amplitude due to the mismatch, whereas the second term in the delta function represents a pixel-level shift. 
3.4. Using CSGBP to Improve Performance in the Presence of Mismatch. In order to avoid errors due to dictionary mismatch, the dictionary needs to be created with upsampled positions and range velocity parameters. This high upsampling may not be feasible due to limited storage and computational complexity. We propose to reduce this high upsampling requirement by using a different prior as well as an iterative scheme. The chosen prior is GBP given as [6]

$$
\sigma_{0, i}^{k} \sim(1-p) \delta\left(\sigma_{0, i}^{k}\right)+p \mathcal{N}\left(\mu_{\sigma}, s_{\sigma}^{2}\right),
$$

where $\sigma_{0, i}^{k}$ is the $i$ th element of $\sigma_{0}$ moving with $k$ th velocity. The main motivation of using this prior is to utilize a priori information about sparsity and signal strength for image reconstruction. $\sigma_{0}$ can be assumed as $q$-sparse that is represented by the probability of active elements $p=q / N_{t}$ in $\sigma_{0}$. The prior assumes that the probability of active elements, that is, an entry of $\boldsymbol{\sigma}_{\mathbf{0}}$ being nonzero, is given by $p$ and these active elements are represented by a Gaussian distribution with mean $\mu_{\sigma}$ and variance $s_{\sigma}^{2}$. The probability of an inactive element is given by $1-p$. The solution to recover $\sigma_{0}$ from $\mathbf{s}_{\mathbf{0}}$ for the prior can be obtained by rewriting (3) as follows:

$$
\mathbf{s}_{0}=\Phi \Psi_{0} \operatorname{diag}\left(\mathbf{z}_{0}\right) \sigma_{0}+\varepsilon,
$$

where $\mathbf{z}_{\mathbf{0}}=\left[z_{0,1}^{1} \cdots z_{0, N}^{1} \cdots z_{0,1}^{N_{v_{x}}} \cdots z_{0,1}^{N_{v_{x}}}\right]^{T}$. The $n$th entry of $\mathbf{z}_{\mathbf{0}}$ is 1 if the corresponding entry in $\sigma_{0}$ is 1 . In this case, $\sigma_{0}$ can be recovered from $\mathbf{s}_{\mathbf{0}}$ in two steps as follows.

(1) The 1st step is the solution to the following problem:

$$
\begin{aligned}
\widehat{\mathbf{z}}_{\mathbf{0}} & =\underset{\mathbf{z}_{\mathbf{0}}}{\operatorname{argmax}} p\left(\mathbf{z}_{\mathbf{0}} \mid \mathbf{s}_{\mathbf{0}}\right) \\
& =\underset{\mathbf{z}_{\mathbf{0}}}{\operatorname{argmax}} p\left(\mathbf{s}_{\mathbf{0}} \mid \mathbf{z}_{\mathbf{0}}\right) p\left(\mathbf{z}_{\mathbf{0}}\right),
\end{aligned}
$$

where

$$
p\left(\mathbf{z}_{\mathbf{0}}\right)=p^{q}(1-p)^{N_{t}-q}
$$

and $p\left(\mathbf{s}_{\mathbf{0}} \mid \mathbf{z}_{\mathbf{0}}\right)$ is given on the next page. For the sake of convenience, we define $\mathbf{D}=\boldsymbol{\Phi} \Psi_{\mathbf{0}}$ and the covariance matrix is given as $\mathbf{R}=s_{\sigma}^{2} \mathbf{D} \operatorname{diag}\left(\mathbf{z}_{\mathbf{0}}\right) \mathbf{D}^{H}+s_{\varepsilon}^{2} \mathbf{I}$. The solution can be further written as

$$
\widehat{\mathbf{z}}_{\mathbf{0}}=\underset{\mathbf{z}_{0}}{\operatorname{argmin}}\left\{-\log p\left(\mathbf{s}_{\mathbf{0}} \mid \mathbf{z}_{\mathbf{0}}\right)-p\left(\mathbf{z}_{\mathbf{0}}\right)\right\} .
$$

(2) The solution $\widehat{\mathbf{z}}_{\mathbf{0}}$ obtained from the 1st step is used to recover estimate of $\sigma_{0}$ by using least squares solution given as

$$
\widehat{\boldsymbol{\sigma}}_{\mathbf{0}}=\underset{\boldsymbol{\sigma}_{0}}{\operatorname{argmin}}\left\|\mathbf{s}_{\mathbf{0}}-\boldsymbol{\Phi} \boldsymbol{\Psi}_{\mathbf{0}} \operatorname{diag}\left(\widehat{\mathbf{z}}_{\mathbf{0}}\right) \boldsymbol{\sigma}_{\mathbf{0}}\right\|_{2}^{2},
$$

$$
\begin{aligned}
p\left(\mathbf{s}_{\mathbf{0}} \mid \mathbf{z}_{\mathbf{0}}\right) & \\
= & \frac{1}{\operatorname{det}(\mathbf{R}) \pi^{M}} \\
& \times \exp \left\{-\left(\mathbf{s}_{\mathbf{0}}-\mu_{\sigma} \mathbf{D} \mathbf{z}_{\mathbf{0}}\right)^{H} \mathbf{R}^{-1}\left(\mathbf{s}_{\mathbf{0}}-\mu_{\sigma} \mathbf{D} \mathbf{z}_{\mathbf{0}}\right)\right\} .
\end{aligned}
$$

Furthermore, this model is suitable for man-made moving scatterers as they may be represented as consisting of a coherent mean part and variation of reflectivities can be represented by an incoherent part represented as variance; that is, $\boldsymbol{\sigma}_{\mathbf{0}} \sim \mathcal{N}\left(\mu_{\sigma}, s_{\sigma}^{2}\right)$. In addition, noise $\boldsymbol{\varepsilon}$ can be assumed to be zero-mean Gaussian with variance $s_{\varepsilon}^{2}$; that is, $\boldsymbol{\varepsilon} \sim \mathcal{N}\left(0, s_{\varepsilon}^{2}\right)$. This CSGBP model can be solved using the algorithms in [6] or [7]. In [6], the raw data is correlated with each column of the matrix $\mathbf{D}$, and the presence or the absence of an element is decided by hypothesis testing. This testing is based on the assumption that the signal is distributed according to the GBP and the noise has Gaussian distribution. In [7], an efficient method is proposed for finding a combination of active and inactive elements.

3.5. Analysis of CSGBP and CSLP Performance in the Presence of Dictionary Mismatch Using Cramer-Rao Bounds. To show theoretically the advantage gained by using CSGBP reconstruction model given in (17) over CSLP model in (7), CRB of the vector $\widehat{\boldsymbol{\sigma}}_{\mathbf{0}}$ estimated from data vector $\mathbf{s}_{\mathbf{0}}$ is calculated as the inverse of Fisher information matrix (FIM) J. We consider $\Phi$ to be identity matrix in (3) for the sake of convenience. The FIM bounds the estimation error in the following form:

$$
E\left[\left(\boldsymbol{\sigma}_{\mathbf{0}}-\widehat{\boldsymbol{\sigma}}_{\mathbf{0}}\right)\left(\boldsymbol{\sigma}_{\mathbf{0}}-\widehat{\boldsymbol{\sigma}}_{\mathbf{0}}\right)^{T}\right] \geq \mathbf{J}^{-1}
$$

$\Phi$ is assumed to be an identity matrix for the sake of convenience. $\mathbf{J}$ is decomposed into two parts [34];

$$
\mathbf{J}_{\mathbf{D}}=E_{\mathbf{s}_{0}, \sigma_{0}}\left[-\frac{\partial^{2}\left\{\log p\left(\mathbf{s}_{\mathbf{0}} \mid \boldsymbol{\sigma}_{\mathbf{0}}\right)\right\}}{\partial \boldsymbol{\sigma}_{\mathbf{0}}{ }^{2}}\right]
$$

and $\mathbf{J}_{\mathbf{P}}$ represents prior information matrix whose individual elements are given as

$$
\mathbf{J}_{\mathbf{P}}=E_{\mathbf{s}_{0}, \boldsymbol{\sigma}_{0}}\left[-\frac{\partial^{2}\left\{\log p\left(\boldsymbol{\sigma}_{\mathbf{0}}\right)\right\}}{\partial \boldsymbol{\sigma}_{\mathbf{0}}{ }^{2}}\right] .
$$

Making use of the explanation given in [35] and smooth approximation; that is, $\left\|\sigma_{0}\right\|_{1} \approx \sum_{i=1}^{N}\left(\left|\sigma_{0, i}\right|^{2}+\varepsilon\right)^{1 / 2}$, the FIM is given as

$$
\mathrm{J}_{\mathrm{LP}}=\left(\frac{\boldsymbol{\Psi}_{\mathbf{0}}{ }^{H} \boldsymbol{\Psi}_{\mathbf{0}}}{s_{\varepsilon}^{2}}\right)
$$

for the case where CSLP is used. When CSGBP is used, the FIM is

$$
\mathbf{J}_{\mathrm{GBP}}=\left(\frac{\boldsymbol{\Psi}_{\mathbf{0}}{ }^{H} \boldsymbol{\Psi}_{\mathbf{0}}}{s_{\varepsilon}^{2}}+\frac{1-p}{s_{\sigma}^{2}}\right) .
$$

As (50) contains more information compared to (49), $\mathbf{J}_{\text {CSGBP }}$ in (50) will be larger and hence the estimation error will be lower that shows the improvement in performance. In case of a dictionary mismatch, using (18) and (19), (47) and (48) become

$$
\widetilde{\mathbf{J}}_{\mathbf{D}}=\boldsymbol{\Psi}^{H} \boldsymbol{\Psi}_{\mathbf{0}} E_{\mathbf{s}_{0}, \boldsymbol{\sigma}_{0}}\left[-\frac{\partial^{2}\left\{\log p\left(\mathbf{s}_{\mathbf{0}} \mid \boldsymbol{\sigma}_{\mathbf{0}}\right)\right\}}{\partial \boldsymbol{\sigma}_{\mathbf{0}}{ }^{2}}\right]
$$


and $\mathbf{J}_{\mathbf{P}}$ represents prior information matrix whose individual elements are given as

$$
\widetilde{\mathbf{J}}_{\mathbf{P}}=\Psi^{H} \Psi_{0} E_{\mathbf{s}_{0}, \boldsymbol{\sigma}_{0}}\left[-\frac{\partial^{2}\left\{\log p\left(\boldsymbol{\sigma}_{\mathbf{0}}\right)\right\}}{\partial \boldsymbol{\sigma}_{0}^{2}}\right] .
$$

Equation (49) becomes

$$
\widetilde{\mathbf{J}}_{\mathrm{LP}}=\left(\frac{\left(\boldsymbol{\Psi}^{H} \boldsymbol{\Psi}_{\mathbf{0}}\right)\left(\boldsymbol{\Psi}_{\mathbf{0}}{ }^{H} \boldsymbol{\Psi}_{\mathbf{0}}\right)}{s_{\varepsilon}^{2}}\right)
$$

and (50) is rewritten as

$$
\widetilde{\mathbf{J}}_{\mathrm{GBP}}=\left(\frac{\left(\boldsymbol{\Psi}^{H} \boldsymbol{\Psi}_{\mathbf{0}}\right)^{H}\left(\boldsymbol{\Psi}_{\mathbf{0}}{ }^{H} \boldsymbol{\Psi}_{\mathbf{0}}\right)}{s_{\varepsilon}^{2}}+\frac{(1-p)\left(\boldsymbol{\Psi}^{H} \boldsymbol{\Psi}_{\mathbf{0}}\right)}{s_{\sigma}^{2}}\right) .
$$

When no dictionary mismatch is present, $\boldsymbol{\Psi}^{H} \boldsymbol{\Psi}_{\mathbf{0}}$ has a maximum value along the diagonal elements. In case of mismatch, the diagonal elements of $\boldsymbol{\Psi}^{H} \boldsymbol{\Psi}_{\mathbf{0}}$ decrease. Subsequently, J decreases leading to an increase in estimated error. It can be inferred that, due to the prior information in (54), the increase of estimated error in the presence of dictionary mismatch is less when CSGBP is used. This can be seen in Figure 1, where an identity matrix of size $512 \times 512$ pixels is used as $\Psi_{\mathbf{0}}$. $\Psi$ is a mismatched basis that is decorrelated with $\Psi_{\mathbf{0}}$ in varying proportions as follows:

$$
\Psi=\rho \Psi_{0}+\sqrt{1-\rho^{2}} \mathcal{N}(0,1),
$$

where $\rho$ is the degree of correlation and the measure $1-\rho$ can be seen equivalent to dictionary mismatch proportion. MSE is calculated using the expression

$$
\mathrm{MSE}_{\mathrm{LP} / \mathrm{GBP}}=\frac{1}{512} \operatorname{trace}\left(\mathrm{J}_{\mathrm{LP} / \mathrm{GBP}}{ }^{-1}\right) \text {. }
$$

It can be seen that using the model given in (39) lowers MSE that can help in countering effects of decorrelation arising due to dictionary mismatch.

3.6. Dealing with Range Velocity Mismatch Using Iterative CSGBP. As outlined in the previous section, CSGBP can compensate for some mismatch, which can help in reducing upsampling requirements. However, it is still not possible to deal with range velocity mismatch using only CSGBP. In general, CS SAR moving target imaging is very sensitive to range velocity mismatch. To avoid any error due to range velocity mismatch, the dictionary should be created with a very high resolution in range velocity; for example, for typical SAR configurations, this resolution can be of the order of $0.01 \mathrm{~m} / \mathrm{s}$. Such a high upsampling requirement is not feasible due to limited memory requirements and very high computations.

In this section, we propose to compensate for velocity mismatch by creating a dictionary iteratively, with range velocities varying at each iteration. In order to reduce

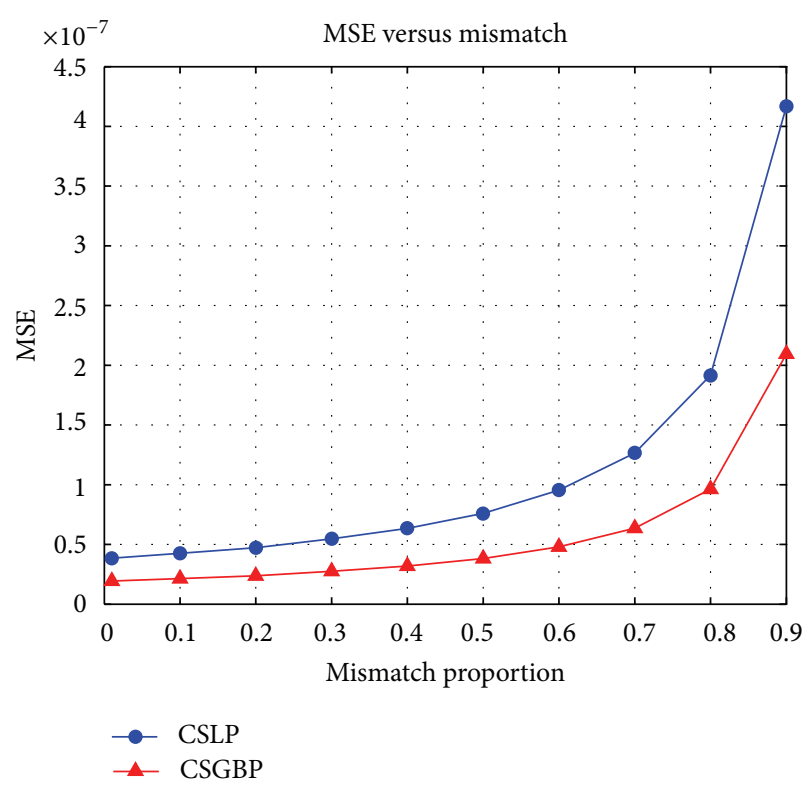

Figure 1: MSE using CSLP and CSGBP.

the computational time, we make use of the following approximation to (6):

$$
d_{n}^{k}\left(\tau_{m}\right) \approx r_{n}-v_{r, n}^{k}\left(\tau_{m}-\frac{y_{n}}{V}\right)+\frac{\left(\tau_{m}-y_{n} / V\right)^{2}}{2 r_{n}} .
$$

We can make use of this approximation to create $\widetilde{s}_{n}^{k+1}\left(t_{l}, \tau_{m}\right)$ from $\hat{s}_{n}^{k}\left(t_{l}, \tau_{m}\right)$ as follows:

$$
\widehat{s}_{n}^{k+1}\left(t_{l}, \tau_{m}\right) \approx \widehat{s}_{n}^{k}\left(t_{l}, \tau_{m}\right) \exp \left(j k_{c} \Delta v_{r, n}^{k}\left(\tau_{m}-y_{n} / V\right)\right),
$$

where $\Delta v_{r, n}^{k}=v_{r, n}^{k}-v_{r, n}^{k+1}$. This allows us to create a dictionary with varying mismatch iteratively using already computed dictionaries. Using the approximation, we propose the following scheme to reconstruct SAR image in the presence of dictionary mismatch.

(1) Create a dictionary with range and azimuth positions at a subpixel resolution. This resolution is chosen so as to meet the upsampling requirements given by (31) and (32). We chose an upsampling factor of 4 in position, which means that the maximum mismatch that can occur is $1 / 8$ of the pixel size. This process is carried out only once.

(2) Carry out CSGBP reconstruction using the dictionary created in Step 1. Due to the upsampling chosen in the range and azimuth directions, and, due to the fact that the range velocity mismatch does not affect the range position, the result contains correct range position as well as range velocity. There will be azimuth position displacements due to range velocity that will be compensated in the next steps.

(3) For each set of reconstructed $n_{k}$ points belonging to the same range velocity $v_{x}^{k}$, regenerate new dictionary 
TABLE 3: Simulation parameters.

\begin{tabular}{|c|c|c|c|}
\hline Carrier frequency & $1.3 \mathrm{GHz}$ & Incidence at center & $40^{\circ}$ \\
\hline Chirp rate & $1 \mathrm{GHz} / \mathrm{s}$ & Sensor velocity & $100 \mathrm{~m} / \mathrm{s}$ \\
\hline Pulse duration & $5 \mu \mathrm{s}$ & Sensor height & $2000 \mathrm{~m}$ \\
\hline $\begin{array}{l}\text { Pulse repetition } \\
\text { frequency }\end{array}$ & $140 \mathrm{~Hz}$ & Azimuth aperture & $7^{\circ}$ \\
\hline Azimuth pixel size & $0.7 \mathrm{~m}$ & Range pixel size & $2.7 \mathrm{~m}$ \\
\hline
\end{tabular}

elements at the selected range positions using (58) and a velocity increment of $\left\lfloor\Delta y_{n}^{k}, s_{y}\right\rfloor$.

(4) Step 3 is repeated by incrementing the velocity in steps of $\left\lfloor\Delta y_{n}^{k}, s_{y}\right\rfloor$, until the reconstructed image is judged to be of the best quality for the $n_{k}$ points. As a quality measure, contrast of the reconstructed vector is calculated as follows:

$$
C_{\widehat{\boldsymbol{\sigma}}_{0}}=\frac{\left\langle\widehat{\boldsymbol{\sigma}}_{0}^{2}\right\rangle}{\left\langle\widehat{\boldsymbol{\sigma}}_{0}\right\rangle^{2}}
$$

where $\langle\cdot\rangle$ is the averaging operator.

(5) Steps 3 and 4 are repeated for each velocity in the dictionary where moving points were detected in Step 2.

\section{Numerical and Imaging Results}

This section presents numerical and imaging results. We give examples with MSE calculated for different amounts of mismatch in range, azimuth, and range velocity for SAR data, followed by imaging examples.

4.1. Numerical Results. The simulation parameters for SAR data are given in Table 3 . A scene of size $50 \mathrm{~m} \times 50 \mathrm{~m}$ or 12 $\times 70$ pixels in range and azimuth directions is considered. Raw data corresponding to multiple points are simulated and $5 \%$ of range data are retained. Positions and amplitudes of these points are chosen randomly, whereas ground-range velocities are chosen randomly from a set of 7 velocities: $\{0,3,4,5,-3,-4,-5\} \mathrm{m} / \mathrm{s}$. Performance in terms of dictionary mismatch is compared. For this purpose, data are generated using a dictionary $\Psi_{0}$ and CS reconstruction is carried out using a mismatched dictionary $\Psi$. The mismatch has a value of 0.01 , followed by values from 0.1 to 0.7 with a step-size of 0.1 . For range and azimuth pixels, the mismatch unit is pixel size, whereas, for range velocity, it is $\mathrm{m} / \mathrm{s}$. A series of simulation is carried out at a signal-to-clutter ratio (SCR) of $20 \mathrm{~dB}$ with randomly chosen positions and velocities of the moving targets. Reconstruction is carried out using CSLP and CSGBP and the resulting MSE between the original points and the reconstructed points are shown in Figure 2. MSE is calculated as follows:

$$
\text { MSE }=\frac{1}{N_{t}} \sum_{n=1}^{N_{t}}\left|\sigma_{0}(n)-\widehat{\sigma}_{0}(n)\right|^{2}
$$

Four main parameters are used in CSGBP reconstruction: $q$, $\mu_{\sigma}, s_{\sigma}$, and $s_{\varepsilon}$, which are initially estimated by using a-priori information. The value of $q$ is decided according to the ratio of supposed active scatterers to total number of scatterers present in the data, whereas the values of $\mu_{\sigma}, s_{\sigma}$, and $s_{\varepsilon}$ are chosen based on SCR. They are then refined by trial and error to get the best results. In general, higher than required values of $q, \mu_{\sigma}$, and $s_{\sigma}$ help in producing weak scatterers but lead to more side lobes, whereas a higher value of $s_{\varepsilon}$ suppresses weak scatterers. From Figure 2, the following observations can be made.

(i) In general, reasonable reconstruction is obtained when the effect of basis mismatch is less than $1 / 3$ of a pixel size.

(ii) MSE is less in case of no range and azimuth pixel mismatch using CSGBP. Similarly, for a small mismatch in range and azimuth directions, the MSE level in case of CSGBP based reconstruction is less. Specifically, it can be remarked that although for the velocity mismatch, MSE increases when velocity mismatch reaches $0.1 \mathrm{~m} / \mathrm{s}$; however, in case of range and azimuth pixels mismatch, MSE is very small as long as pixel mismatch stays less than 0.3 of the pixel size. Thus, CSGBP can be used for better reconstruction and reduction of the dictionary size in practical scenarios, compared to CSLP based reconstruction, where the MSE is higher even in case of no dictionary mismatch.

(iii) MSE for range velocity is high using both methods. After the mismatch of $0.1 \mathrm{~m} / \mathrm{s}$, CSLP seems to give slightly lower MSE. The reason may be that CSGBP gives higher number of side lobes. Further simulations for the values of mismatch ranging from 0.01 to 0.1 in a step size of $0.01 \mathrm{~m} / \mathrm{s}$ are shown in Figure 3 . It can be seen that MSE using CSGBP is still smaller than that using CSLP. The reason for not reporting any ill effects of mismatch in velocity in [25] may be that the amount of mismatch considered is small for the configuration that was studied. There are two types of moving targets that are considered in [25], a slow one and a fast one. The former target has a range velocity of $2.35 \mathrm{~m} / \mathrm{s}$, whereas the latter target has a range velocity of $28.15 \mathrm{~m} / \mathrm{s}$. The range velocity mismatch for the slow target is $0.85 \mathrm{~m} / \mathrm{s}$, whereas, for the fast target, it is $0.45 \mathrm{~m} / \mathrm{s}$. The amount of mismatch is small to have any effect on the reconstruction for the particular case. This can be seen from reconstruction results in Figure 5 of [25] that shows focussing assuming no motion. The slow object, despite having a mismatch of $2.35 \mathrm{~m} / \mathrm{s}$ in the range direction, is still focussed at the same position. Our results show theoretically as well as experimentally that a mismatch in velocity can have a serious impact on reconstruction.

(iv) The error increases gradually for position mismatch but increases very rapidly for range velocity mismatch. The reason is that, in case of range velocity mismatch, a large shift arises in azimuth direction. This is due to the reason that $r_{n}$ is of the order of $10^{3} \mathrm{~m}$; 


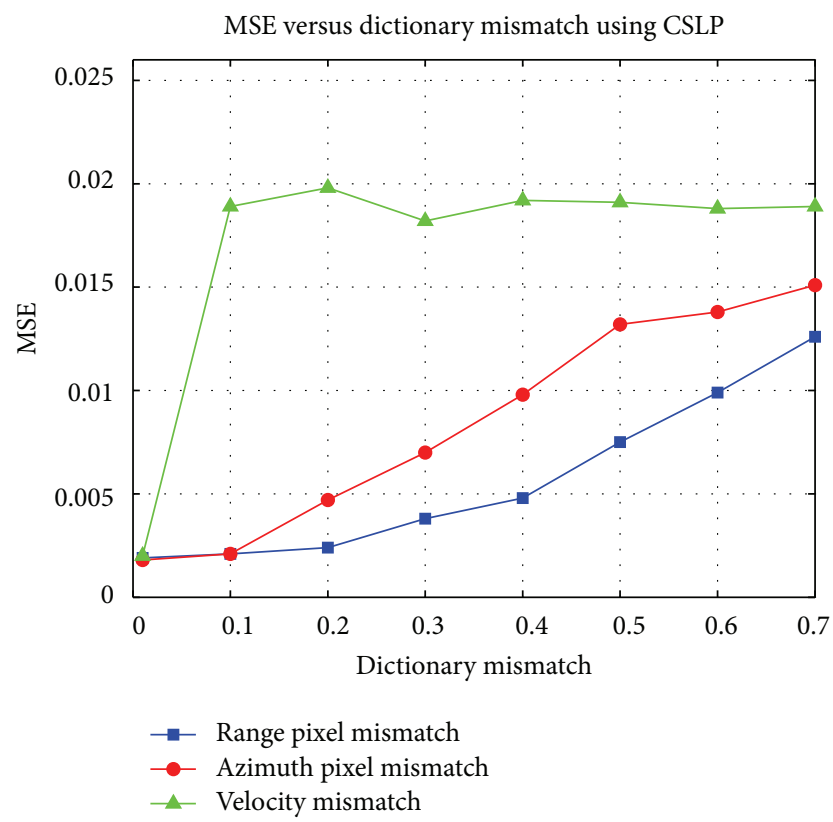

(a) MSE versus dictionary mismatch using CSLP

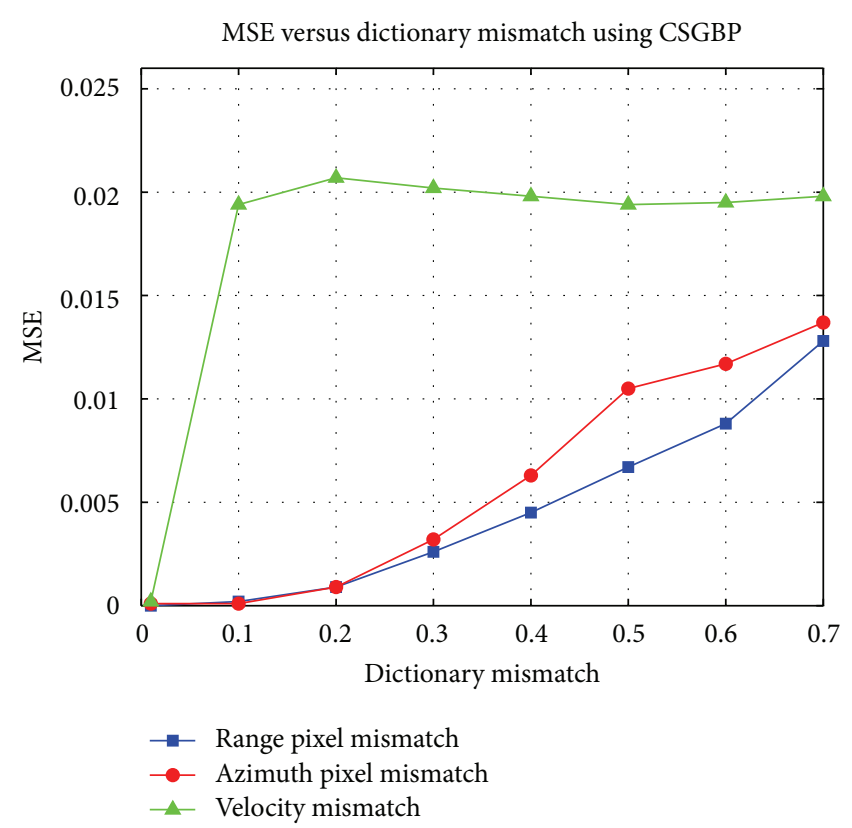

(b) MSE versus dictionary mismatch using CSGBP

FIGURE 2: MSE versus dictionary mismatch using CSLP and CSGBP.

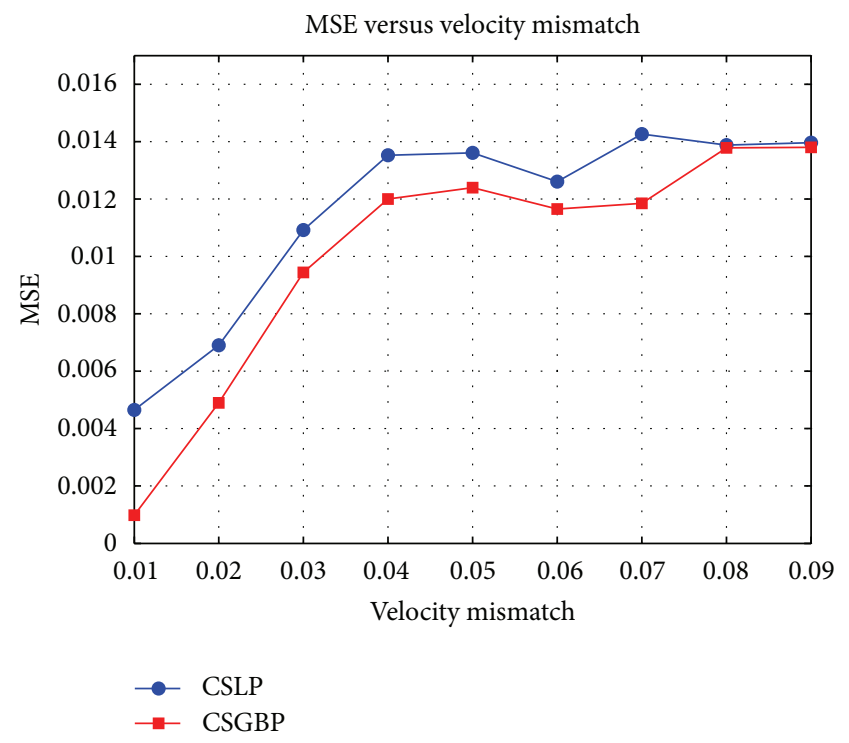

FIGURE 3: MSE for range velocity mismatch using CSLP and CSGBP.

for example, for a velocity mismatch of $0.05 \mathrm{~m} / \mathrm{s}$, $r_{n}=2595 \mathrm{~m}$, and $\theta_{n}=40^{\circ}$, there is a single-pixel shift between the original and reconstructed position. Thus, the reconstruction result will contain azimuth pixels shifted according to the mismatch, which leads to a sudden increase in MSE. As there is a total misalignment between actual and estimation positions, MSE rises and stays at a roughly constant maximum level. This is further demonstrated in Figure 4, where a reconstructed scene contains a single pixel shift with

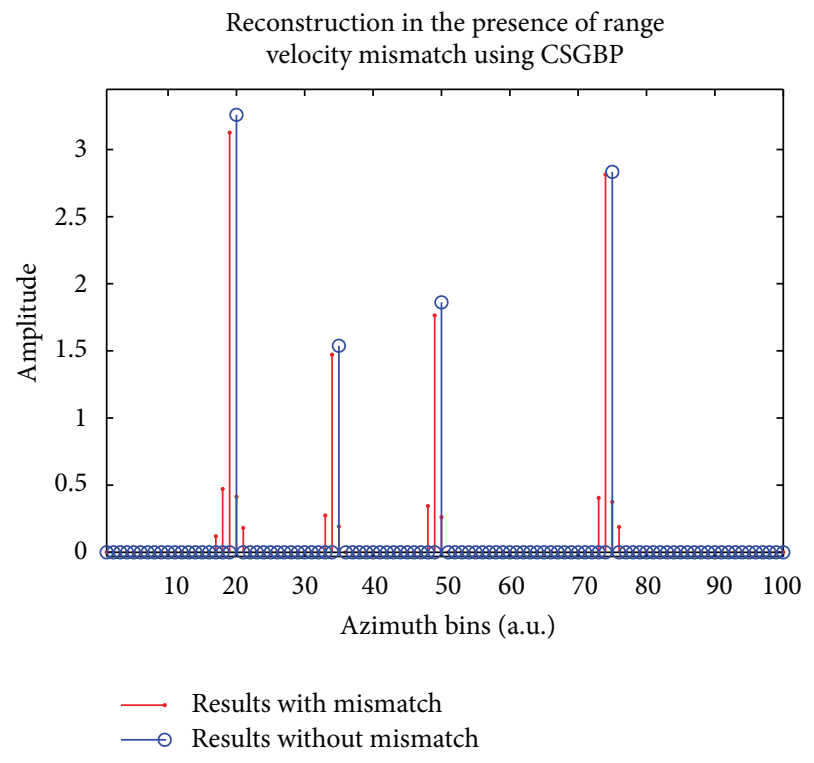

FIGURE 4: Effects of range velocity mismatch using CSGBP. A singlepixel shift and some side lobes can be seen.

respect to the actual position. The reason is that there is a mismatch in range velocity of $0.05 \mathrm{~m} / \mathrm{s}$. A loss of amplitude and side lobes can be seen.

(v) The error in azimuth is more than that in range position in general, especially using CSLP. The reason is that there are more than one combination of $r_{n}, y_{n}$, and $\theta_{n}$ that lead to closely resembling values of $r_{m}$ and $y_{m}$ in (26); for example, for the parameters given in Table 3, we can see that $r_{n}$ and $y_{n}$ of $2594.5 \mathrm{~m}$ and 
Reconstruction in the presence of azimuth pixel mismatch using CSLP

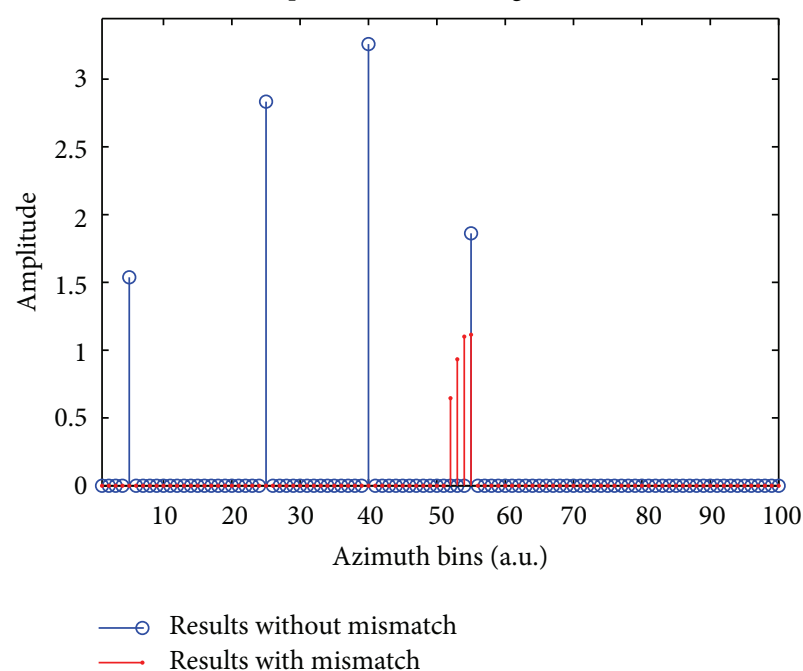

(a) Effects of azimuth pixel mismatch using CSLP. Only one position is identified correctly with a velocity of $3 \mathrm{~m} / \mathrm{s}$

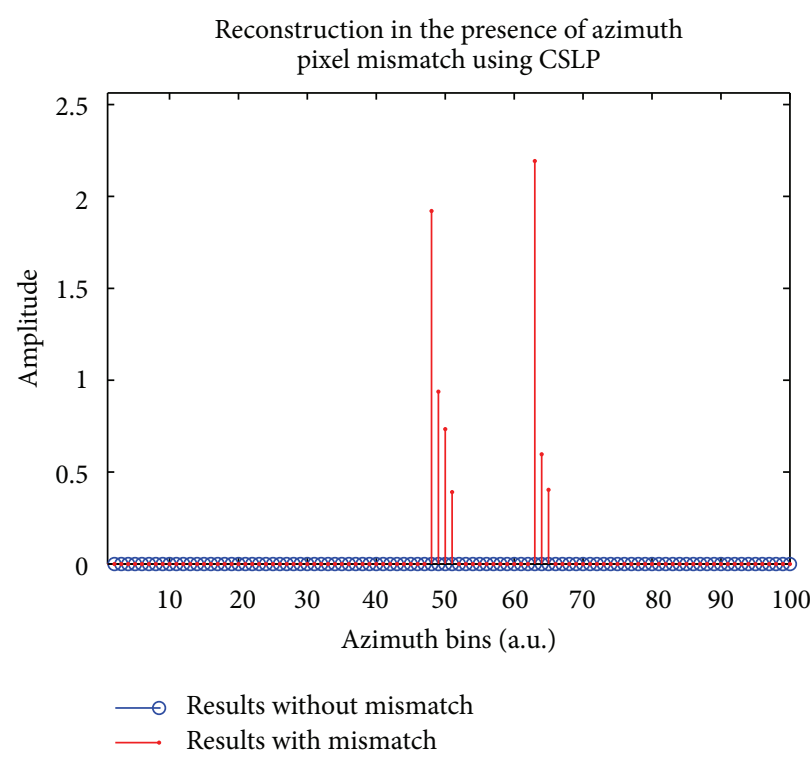

(b) Effects of azimuth pixel mismatch using CSLP. The scatterers are identified at a velocity of $4 \mathrm{~m} / \mathrm{s}$, instead of a velocity of $3 \mathrm{~m} / \mathrm{s}$. Scatterers are also shifted from their true positions

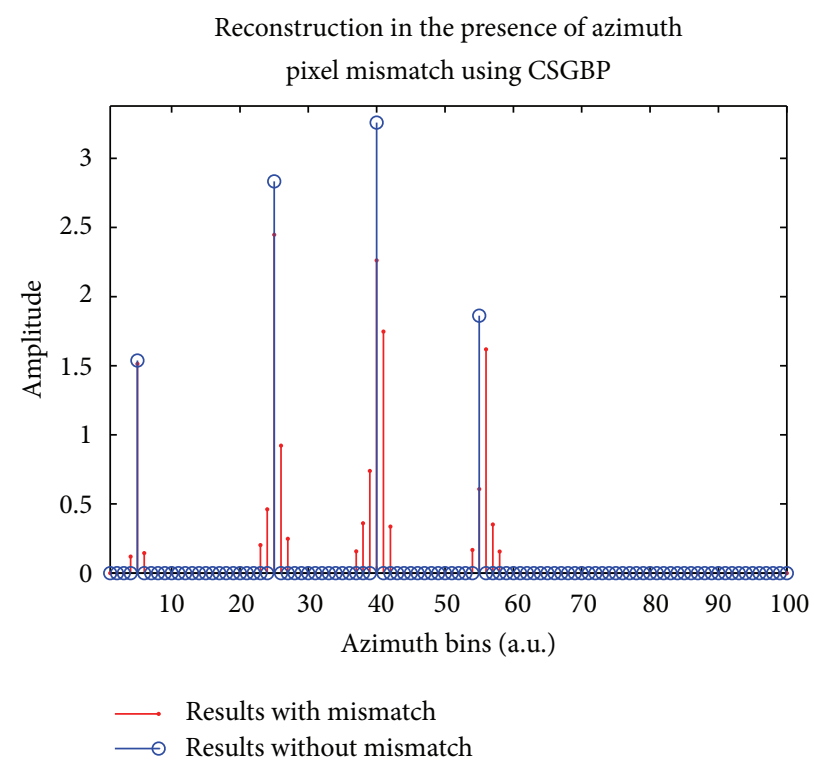

(c) Effects of azimuth pixel mismatch using CSGBP. The positions are identified correctly with the presence of higher side lobes

FIGURE 5: Effects of azimuth pixel mismatch using CSLP and CSGBP.

$-25.7143 \mathrm{~m}$ with $v_{x}=3 \mathrm{~m} / \mathrm{s}$ lead to $r_{m}$ and $y_{m}$ of $2594 \mathrm{~m}$ and $-75.2693 \mathrm{~m}$. The same values of $r_{m}$ and $y_{m}$ are obtained with similar value of $r_{n}$ and $y_{n}=$ $-9.1154 \mathrm{~m}$ with $v_{x}=4 \mathrm{~m} / \mathrm{s}$ as well as $y_{n}=7.2128 \mathrm{~m}$ and $v_{x}=5 \mathrm{~m} / \mathrm{s}$. Thus, it is possible that a dictionary mismatch will lead to selection of dictionary elements and subsequently, side lobes that are not in the immediate neighborhood. This is demonstrated in Figure 5, where there are 4 points at different azimuth positions having a velocity of $3 \mathrm{~m} / \mathrm{s}$. The mismatch is $0.1,0.3,0.5$, and 0.7 of a pixel size. When CSLP is used to carry out reconstruction, only a single point is identified with a velocity of $3 \mathrm{~m} / \mathrm{s}$. This is shown in Figure 5(a). Two of the points are detected at shifted azimuth positions with a velocity of $4 \mathrm{~m} / \mathrm{s}$, as shown in Figure 5(b). The fourth point is not identified at all. In case of reconstruction using CSGBP, all of the four points are identified correctly as shown in Figure 5(c), albeit with higher side lobes. This also demonstrates the advantage offered by CSGBP by identifying correct positions and velocity even in the presence of pixel mismatch. 


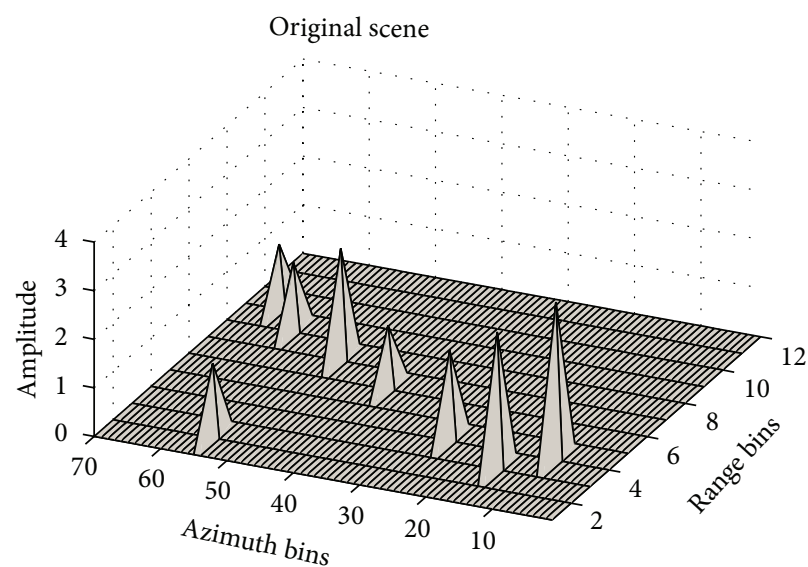

(a) Original scene used for showing effects of range and azimuth pixels mismatch

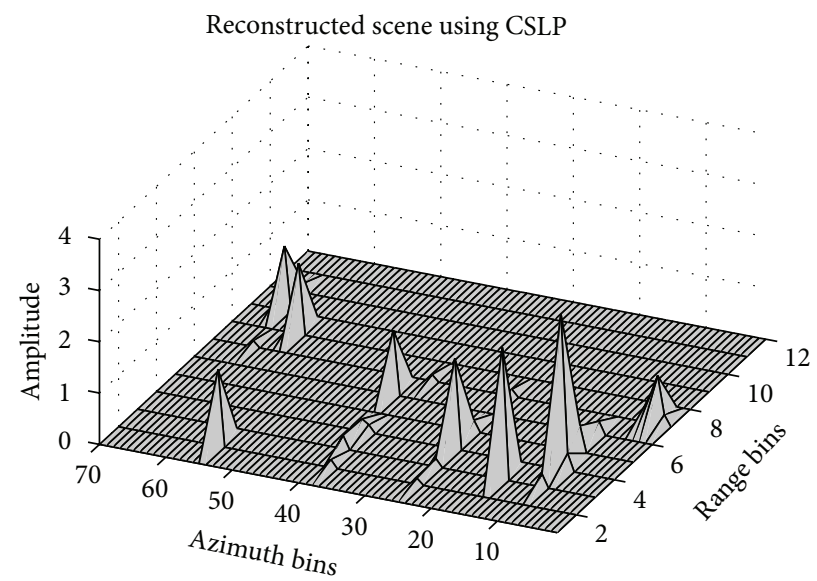

(b) Reconstruction using CSLP. One of the scatterers is not identified correctly

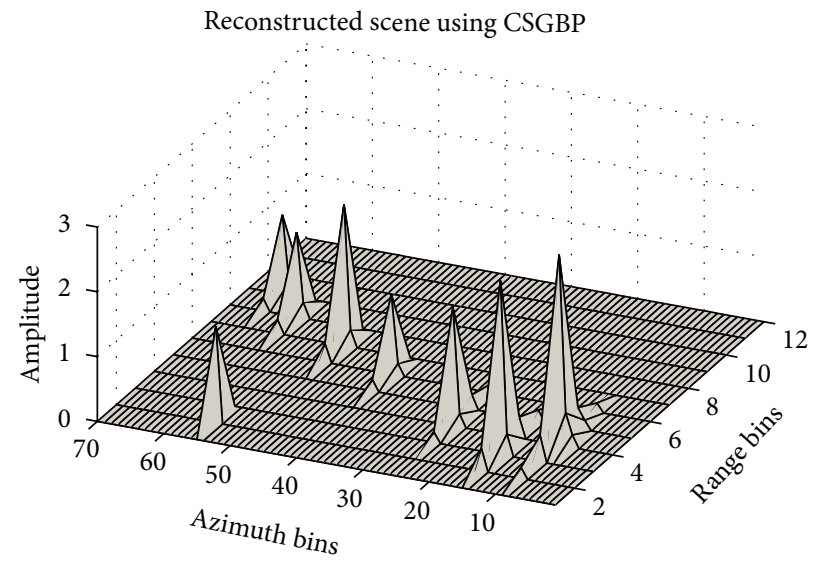

(c) Reconstruction using CSGBP. All positions are identified correctly

FIGURE 6: Reconstruction in the presence of range and azimuth pixels mismatch using CSLP and CSGBP.

4.2. Imaging Results. In this section, we compare the reconstruction performance of CSLP and CSGBP through imaging results and demonstrate the effectiveness of the proposed iterative CSGBP. Eight points are simulated at positions (2, $55),(3,15),(4,9),(4,25),(6,40),(7,50),(8,60)$, and $(9$, 65). The scene is shown in Figure 6(a). The velocities of the points are $3,4,-5,5,4,-3,-4$, and $-5 \mathrm{~m} / \mathrm{s}$, respectively. Reconstruction is carried out in the presence of a mismatch of $1 / 8$ of a pixel size in range and azimuth. Results using CSLP are shown in Figure 6(b), which shows that the point at $(7,50)$ is not reconstructed correctly. CSGBP results shown in Figure 6(c) indicate that all the points are correctly reconstructed. This demonstrates the superior performance of CSGBP. Furthermore, results using CSGBP show side lobes in the vicinity of actual positions, whereas, in case of CSGBP, the side lobes appear at positions that are not in the vicinity of actual positions. A further example is shown with a mismatch of $0.4 \mathrm{~m} / \mathrm{s}$ in range velocity. The original scene is shown in Figure 7(a), where there are closely spaced scatterers roughly in the middle of the scene. They have a velocity of $4.4 \mathrm{~m} / \mathrm{s}$, whereas the closest velocity in the dictionary is $4 \mathrm{~m} / \mathrm{s}$. Reconstruction using both CSLP and CSGBP shows shifted results due to the mismatch. Furthermore, results obtained using CSLP were obtained at a velocity of $5 \mathrm{~m} / \mathrm{s}$. Result obtained using iterative CSGBP is shown in Figure $7(\mathrm{~d})$, where the points are located at their correct positions. The velocity in the dictionary is increased iteratively with a step size of $0.05 \mathrm{~m} / \mathrm{s}$, until the highest contrast is achieved. A plot of contrast with velocity is shown in Figure 8, where it can be seen that the contrast is the highest when the velocity in the dictionary matches the actual velocity. This shows that creating dictionary elements iteratively and using contrast to measure quality are effective methods for dealing with CS moving target imaging in the presence of range velocity mismatch. Another example is shown with a scene in Figure 9(a). The points are at positions of $(2,5),(5,45)$, $(6,34),(2,70),(10,15)$, and $(7,65)$. The point at $(2,5)$ has a velocity of $-4.9 \mathrm{~m} / \mathrm{s}$, the point at $(5,45)$ has a velocity of $-4 \mathrm{~m} / \mathrm{s}$, and the point at $(6,34)$ has a velocity of $-3 \mathrm{~m} / \mathrm{s}$. The remaining points have a velocity of $3.3 \mathrm{~m} / \mathrm{s}$. Thus, there is a mismatch of $0.1 \mathrm{~m} / \mathrm{s}$ and $0.3 \mathrm{~m} / \mathrm{s}$. The pixel at position $(2,5)$ has a $1 / 2$ pixel mismatch in azimuth and $1 / 4$ pixel mismatch in azimuth. The pixel at positions $(6,34)$ has a $1 / 4$ pixel mismatch in azimuth and 1/2 pixel mismatch in range. 


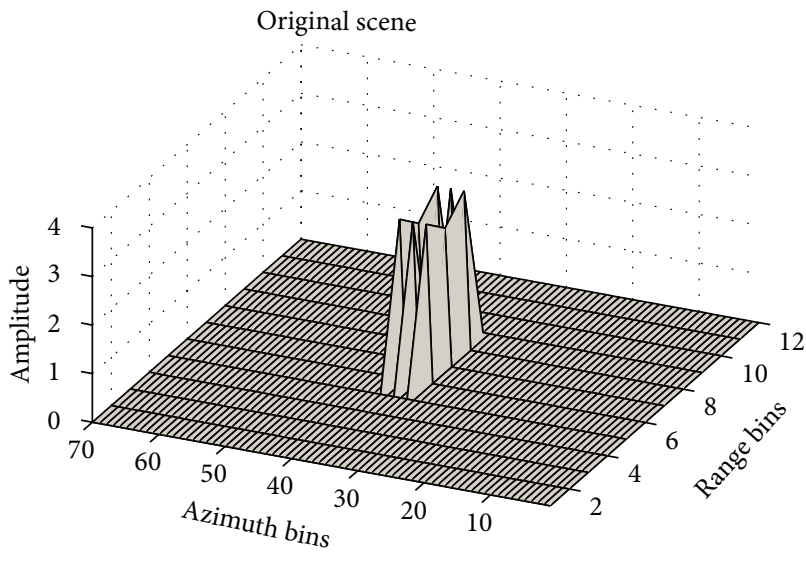

(a) Original scene for showing effects of range velocity mismatch

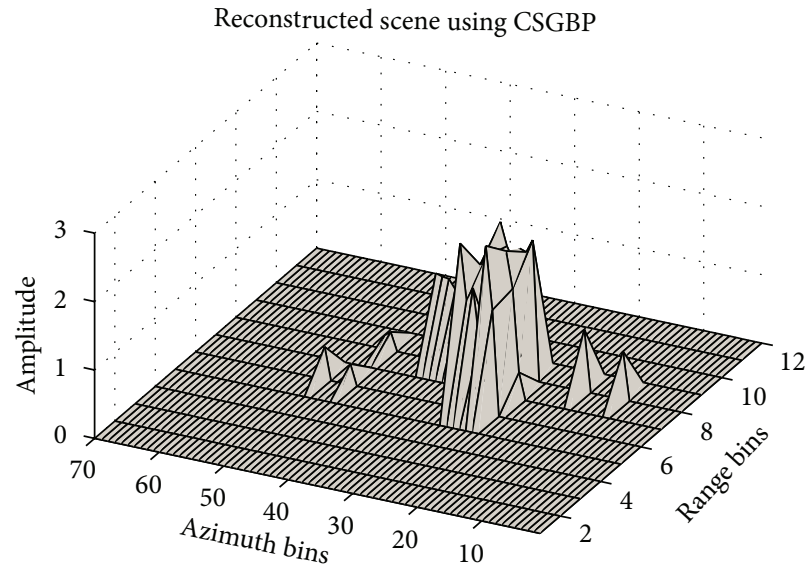

(c) Reconstruction using CSGBP. The points are shifted in azimuth direction

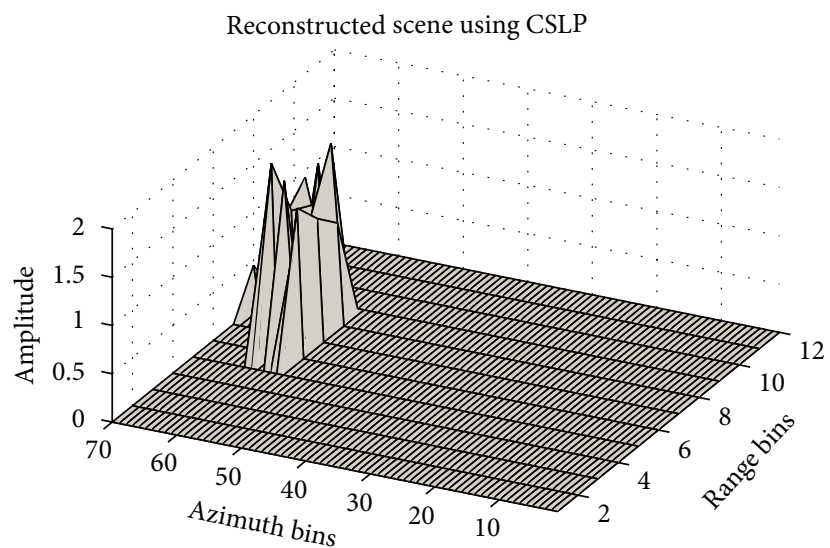

(b) Reconstruction using CSLP. The points are shifted in azimuth direction Reconstructed scene using CSGBP

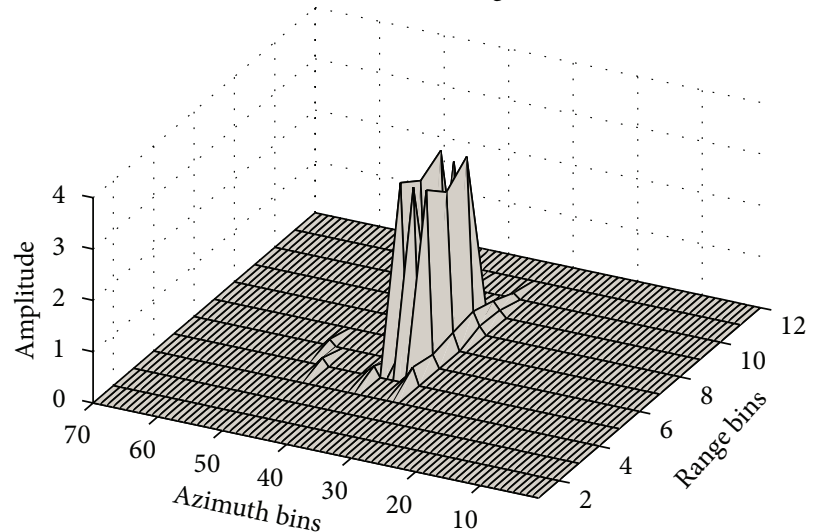

(d) Reconstruction using iterative CSGBP. The points are at their original positions. A few side lobes can be seen.

FIgURE 7: Reconstruction using CSLP, CSGBP, and iterative CSGBP in the presence of range velocity mismatch.

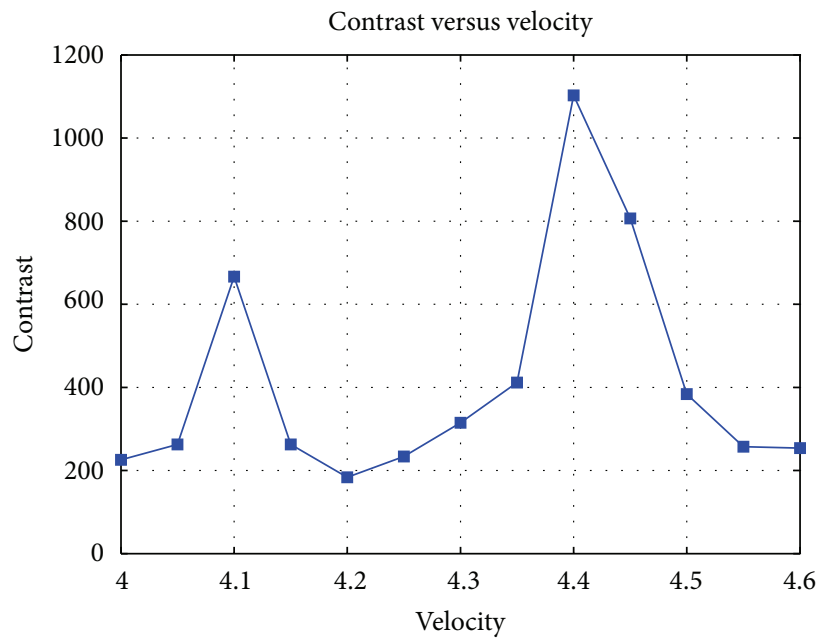

Figure 8: Contrast versus velocity. The velocity of the point is $4.4 \mathrm{~m} / \mathrm{s}$. 


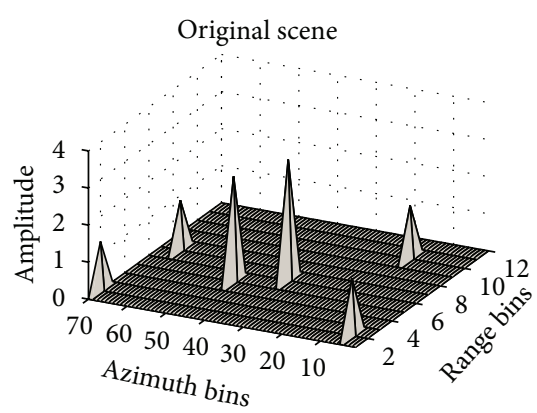

(a) Original scene used for showing effects of range pixel, azimuth pixel, and range velocity mismatch

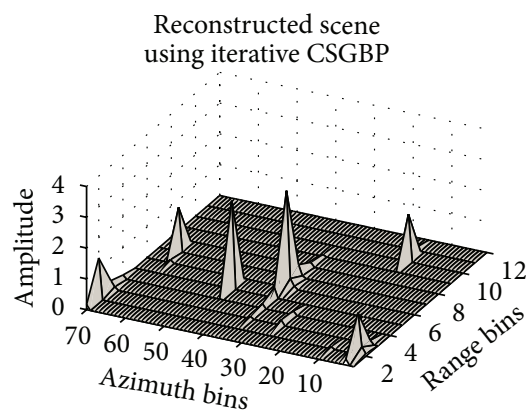

(d) Reconstruction using iterative CSGBP. Points moving at $3.3 \mathrm{~m} / \mathrm{s}$ are focussed

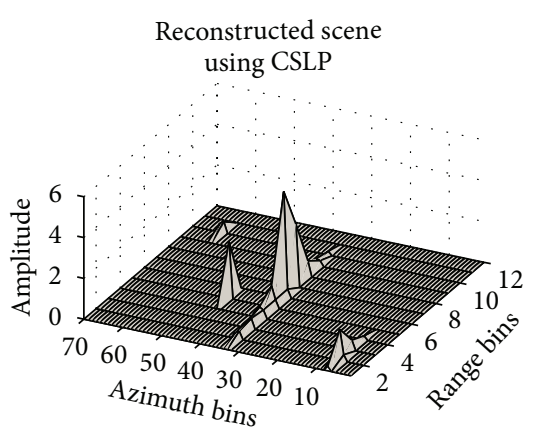

(b) Reconstruction using CSLP. Points at $(7,65)$ and $(2,70)$ are not identified

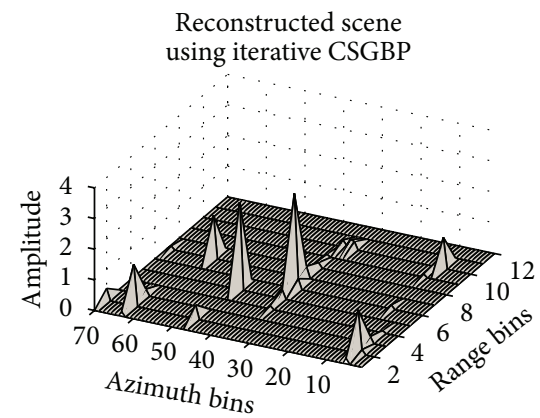

(e) Reconstruction using iterative CSGBP. Point moving at $-4.9 \mathrm{~m} / \mathrm{s}$ is focussed

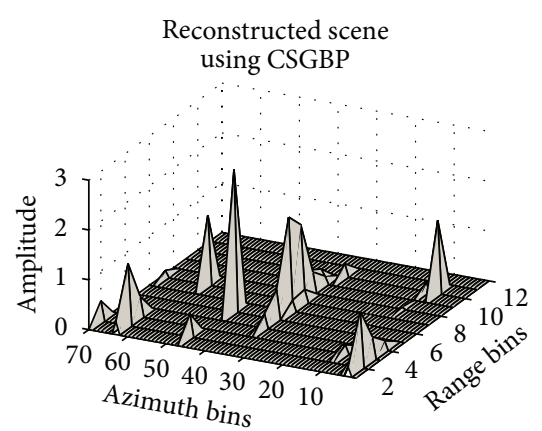

(c) Reconstruction using CSGBP. All range positions are correctly identified

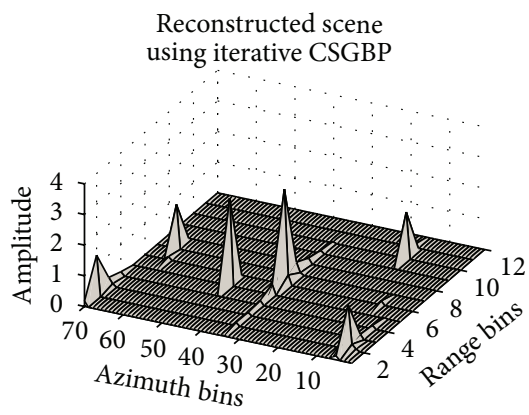

(f) Reconstruction using iterative CSGBP. All points are focussed

FIgURE 9: Reconstruction using CSLP, CSGBP, and iterative CSGBP.

CSLP reconstruction results with the dictionary containing elements at $1 / 4$ pixel spacing are shown in Figure 9(b). CSLP is unable to detect two of the scatterers at positions $(7,65)$ and $(2,70)$; other scatterers having velocity mismatch are shifted in azimuth. CSGBP reconstruction results are shown in Figure 9(c). All the range positions are correctly identified, but the result is shifted in azimuth. Result using iterative CSGBP with velocity varying in a step size of $0.05 \mathrm{~m} / \mathrm{s}$ for each velocity in the dictionary is shown in Figures 9(d)-9(f). The result obtained by maximizing the contrast for the point at $(6,34)$ is shown in Figure $9(\mathrm{~d})$, where the points moving at $3.3 \mathrm{~m} / \mathrm{s}$ are focussed at their true position. Some side lobes can be seen. Similarly, the point moving at $-4.9 \mathrm{~m} / \mathrm{s}$ is shown correctly focussed in Figure 9(e). Final result obtained using the calculated velocities is shown in Figure 9(f), where all the points are focussed at their true positions. Some side lobes can be observed.

\section{Conclusion}

In this paper, we studied compressed sensing (CS) synthetic aperture radar (SAR) moving target imaging in the presence of dictionary mismatch. We analyzed the sensitivity of the imaging process to range pixel, azimuth pixel, and range velocity mismatches. The mismatch analysis shows that the reconstruction error increases with mismatch and especially increases very rapidly in the presence of range velocity mismatch. Unlike existing references, we show that using a Gaussian-Bernoulli prior compared to the traditionally used Laplacian prior offers advantage in CS SAR imaging for dealing with small mismatch. This advantage is apparent in dealing with positions mismatch. We calculated Cramer-Rao bounds that demonstrate theoretically the lowering of mean square error between actual and reconstructed result by using the GBP. We show that creating an upsampled dictionary and using the GBP for reconstruction can deal with position mismatch. We also presented an iterative scheme to deal with the range velocity mismatch where dictionary elements are created efficiently. CS reconstruction is carried out at each iteration until the image contrast is maximized for each velocity. Numerical and imaging examples confirm the analysis and the effectiveness of the proposed upsampling and iterative scheme.

\section{Notations and Symbols}

$t$ : Range time

$f_{c}$ : Central frequency

$r$ : Slant-range positions

$y$ : Azimuth positions

$k_{c}: 4 \pi f_{c} / c$

$K$ : Chirp rate

$V$ : Sensor velocity

$h$ : Antenna height

$T_{p}$ : Pulse length

$x$ : Ground-range positions 


\begin{tabular}{|c|c|}
\hline$c:$ & Speed of light \\
\hline$\tau:$ & Azimuth time \\
\hline$\theta_{n}:$ & $\begin{array}{l}\text { Incidence angle at range } r_{n} \text {, equal to } \\
\cos ^{-1}\left(h / r_{n}\right)\end{array}$ \\
\hline$v_{x}^{k}:$ & $k$ th ground range velocity \\
\hline$v_{r, n}^{\tilde{k}}:$ & $\begin{array}{l}\text { Translational velocity, equal to } v_{r, n}^{k} \\
v_{x}^{k} \sin \theta_{n}\end{array}$ \\
\hline$s_{r}:$ & Pixel size in range \\
\hline$s_{y}:$ & Pixel size in azimuth \\
\hline$n_{r}:$ & Number of range pixels in the scene \\
\hline$n_{y}:$ & Number of azimuth pixels in the scene \\
\hline$N_{r}:$ & Number of range pixels in raw data \\
\hline$N_{y}:$ & Number of azimuth pixels in raw data \\
\hline$N_{v x}:$ & Number of range velocities \\
\hline$N_{t}:$ & $N \times N_{v x}$ \\
\hline$N:$ & $n_{r} \times n_{y}$ \\
\hline$N_{s}:$ & $N_{r} \times N_{y}$ \\
\hline $\mathrm{s}_{0}$ : & $\begin{array}{l}\text { Raw data from all the points in the scene } \\
\text { arranged in } 1 \mathrm{D} \text { form }\end{array}$ \\
\hline $\mathbf{s}_{n}^{k}$ & $\begin{array}{l}\text { Raw data for } n \text {th point moving with } k \text { th } \\
\text { velocity, arranged in } 1 \mathrm{D} \text { form }\end{array}$ \\
\hline$\widetilde{s}_{n}^{k}\left(t_{m}, \tau_{n}\right)$ & $\begin{array}{l}\text { Raw data element for range time } t_{m} \text { and } \\
\text { azimuth time } \tau_{n}\end{array}$ \\
\hline$d_{n}^{k}\left(\tau_{n}\right)$ & $\begin{array}{l}\text { Radar-target distance for } n \text {th point moving } \\
\text { with } k \text { th velocity }\end{array}$ \\
\hline$\Psi_{0}:$ & Original dictionary \\
\hline$\Psi:$ & Mismatched dictionary \\
\hline$\sigma_{0}:$ & Original reflectivity vector \\
\hline$\sigma:$ & Mismatched reflectivity vector \\
\hline$\widehat{\sigma}_{0}:$ & Reconstructed reflectivity vector \\
\hline$\widehat{\boldsymbol{\sigma}}:$ & $\begin{array}{l}\text { Reconstructed reflectivity vector in the } \\
\text { presence of mismatch }\end{array}$ \\
\hline$\widehat{\Sigma}_{0}:$ & Reconstructed reflectivity in 2D \\
\hline$\Phi:$ & Sampling matrix \\
\hline$M:$ & Number of columns of $\Phi$ \\
\hline$\varepsilon:$ & Noise vector \\
\hline$|\langle\rangle|:$, & Inner product \\
\hline$\langle\cdot\rangle:$ & Averaging operation \\
\hline$\Theta_{n}^{k}:$ & $\begin{array}{l}\text { Rotation angle with which a moving scat- } \\
\text { terer can be seen equivalent to a static } \\
\text { scatterer }\end{array}$ \\
\hline$\Delta r_{n}:$ & Subpixel mismatch in range position \\
\hline$\Delta y_{n}:$ & Subpixel mismatch in azimuth position \\
\hline$\Delta v_{x}^{k}:$ & Fraction of $\mathrm{m} / \mathrm{s}$ mismatch in range velocity \\
\hline$\Delta y_{n}^{\bar{k}}:$ & $\begin{array}{l}\text { Shift in range position due to range velocity } \\
\text { mismatch }\end{array}$ \\
\hline$\sigma_{0, n}^{k}:$ & $\begin{array}{l}\text { Element of reflectivity vector at } n \text {th posi- } \\
\text { tion and moving with } k \text { th velocity }\end{array}$ \\
\hline$\widehat{\sigma}_{0, n}^{k}:$ & $\begin{array}{l}\text { Reconstructed element of reflectivity vec- } \\
\text { tor at } n \text {th position and moving with } k \text { th } \\
\text { vecloity }\end{array}$ \\
\hline$\overline{\Delta r}:$ & Dictionary resolution for range \\
\hline$\widetilde{\Delta y}:$ & Dictionary resolution for azimuth \\
\hline$\Delta v_{x}:$ & Dictionary resolution for range velocity \\
\hline$r_{m}:$ & Range position for equivalent static point \\
\hline$y_{m}:$ & $\begin{array}{l}\text { Azimuth position for equivalent static } \\
\text { point }\end{array}$ \\
\hline
\end{tabular}

p: $\quad$ Probability of active coefficients in $\widehat{\boldsymbol{\sigma}}_{\mathbf{0}}$

$q: \quad$ Sparsity of $\widehat{\boldsymbol{\sigma}}_{0}$

$\rho: \quad$ Correlation

$s_{\varepsilon}^{2}$ : $\quad$ Variance for noise

$s_{\sigma}^{2}$ : $\quad$ Variance of reflectivity vector

I: $\quad$ Identity matrix

$C_{\widehat{\boldsymbol{\sigma}}_{0}}: \quad$ Contrast of $\widehat{\boldsymbol{\sigma}}_{\mathbf{0}}$

FIM: $\quad$ Fisher information matrix

CSLP: CS reconstruction with Laplacian prior

CSGBP: CS reconstruction with GaussianBernoulli prior

$\mathrm{J}_{\mathrm{LP}}: \quad$ FIM for CSLP without mismatch

$\mathrm{J}_{\mathrm{GBP}}: \quad$ FIM for CSGBP without mismatch

$\widetilde{\mathbf{J}}_{\mathrm{LP}}: \quad$ FIM for CSLP with mismatch

$\widetilde{\mathbf{J}}_{\mathrm{GBP}}: \quad$ FIM for CSGBP with mismatch.

\section{References}

[1] E. J. Candès and T. Tao, "Decoding by linear programming," IEEE Transactions on Information Theory, vol. 51, no. 12, pp. 4203-4215, 2005.

[2] E. J. Candès, J. Romberg, and T. Tao, "Robust uncertainty principles: exact signal reconstruction from highly incomplete frequency information," IEEE Transactions on Information Theory, vol. 52, no. 2, pp. 489-509, 2006.

[3] D. L. Donoho, "Compressed sensing," IEEE Transactions on Information Theory, vol. 52, no. 4, pp. 1289-1306, 2006.

[4] J. A. Tropp and A. C. Gilbert, "Signal recovery from random measurements via orthogonal matching pursuit," IEEE Transactions on Information Theory, vol. 53, no. 12, pp. 4655-4666, 2007.

[5] S. Ji, Y. Xue, and L. Carin, "Bayesian compressive sensing," IEEE Transactions on Signal Processing, vol. 56, no. 6, pp. 2346-2356, 2008.

[6] H. Zayyani, M. Babaie-Zadeh, and C. Jutten, "Bayesian pursuit algorithm for sparse representation," in Proceedings of the IEEE International Conference on Acoustics, Speech, and Signal Processing (ICASSP '09), pp. 1549-1552, April 2009.

[7] P. Schniter, L. C. Potter, and J. Ziniel, "Fast bayesian matching pursuit," in Proceedings of the 2008 Information Theory and Applications Workshop (ITA '08), pp. 326-332, San Diego, Calif, USA, February 2008.

[8] J. Ma, "Single-Pixel remote sensing," IEEE Geoscience and Remote Sensing Letters, vol. 6, no. 2, pp. 199-203, 2009.

[9] M. Lustig, D. Donoho, and J. M. Pauly, "Sparse MRI: the application of compressed sensing for rapid MR imaging," Magnetic Resonance in Medicine, vol. 58, no. 6, pp. 1182-1195, 2007.

[10] G. Shi, J. Lin, X. Chen, F. Qi, D. Liu, and L. Zhang, "UWB echo signal detection with ultra-low rate sampling based on compressed sensing," IEEE Transactions on Circuits and Systems II, vol. 55, no. 4, pp. 379-383, 2008.

[11] J. Ma and F.-X. Le Dimet, "Deblurring from highly incomplete measurements for remote sensing," IEEE Transactions on Geoscience and Remote Sensing, vol. 47, no. 3, pp. 792-802, 2009.

[12] X. Nie, D.-Y. Zhu, and Z.-D. Zhu, "Application of synthetic bandwidth approach in SAR polar format algorithm using the deramp technique," Progress in Electromagnetics Research, vol. 80, pp. 447-460, 2008. 
[13] Q. Huang, L. Qu, B. Wu et al., "UWB through-wall imaging based on compressive sensing," IEEE Transactions on Geoscience and Remote Sensing, vol. 48, no. 3, pp. 1408-1415, 2010.

[14] W. Zhang, M. Amin, F. Ahmad et al., "Ultrawideband impulse radar through-the-wall imaging with compressive sensing," International Journal of Antennas and Propagation, vol. 2012, Article ID 251497, 11 pages, 2012.

[15] M. Duman and A. Gurbuz, "Performance analysis of compressive-sensing-based through-the-wall imaging with effect of unknown parameters," International Journal of Antennas and Propagation, vol. 2012, Article ID 405145, 11 pages, 2012.

[16] X. X. Zhu and R. Bamler, "Tomographic SAR inversion by $\mathrm{L}_{1}$ norm regularization-the compressive sensing approach," IEEE Transactions on Geoscience and Remote Sensing, vol. 48, no. 10, pp. 3839-3846, 2010.

[17] X. X. Zhu and R. Bamler, "Super-resolution power and robustness of compressive sensing for spectral estimation with application to spaceborne tomographic SAR," IEEE Transactions on Geoscience and Remote Sensing, vol. 50, no. 1, pp. 247-258, 2012.

[18] A. Budillon, A. Evangelista, and G. Schirinzi, "Threedimensional SAR focusing from multipass signals using compressive sampling," IEEE Transactions on Geoscience and Remote Sensing, vol. 49, no. 1, pp. 488-499, 2011.

[19] M. T. Alonso, P. López-Dekker, and J. J. Mallorquí, "A novel strategy for radar imaging based on compressive sensing," IEEE Transactions on Geoscience and Remote Sensing, vol. 48, no. 12, pp. 4285-4295, 2010.

[20] R. K. Raney, "Synthetic aperture imaging radar and moving targets," IEEE Transactions on Aerospace and Electronic Systems, vol. 7, no. 3, pp. 499-505, 1971.

[21] J. H. G. Ender, “On compressive sensing applied to radar," Signal Processing, vol. 90, no. 5, pp. 1402-1414, 2010.

[22] Q. Wu, M. Xing, C. Qiu, B. Liu, Z. Bao, and T.-S. Yeo, "Motion parameter estimation in the SAR system with low PRF sampling," IEEE Geoscience and Remote Sensing Letters, vol. 7, no. 3, pp. 450-454, 2010.

[23] A. S. Khwaja and J. Ma, "Applications of compressed sensing for sar moving-target velocity estimation and image compression," IEEE Transactions on Instrumentation and Measurement, vol. 60, no. 8, pp. 2848-2860, 2011.

[24] Y. G. Lin, B. C. Zhang, W. Hong, and Y. R. Wu, "Along-track interferometric SAR imaging based on distributed compressed sensing," Electronics Letters, vol. 46, no. 12, pp. 858-860, 2010.

[25] I. Stojanovic and W. C. Karl, "Imaging of moving targets with multi-static SAR using an overcomplete dictionary," IEEE Journal on Selected Topics in Signal Processing, vol. 4, no. 1, pp. 164-176, 2010.

[26] M. A. Herman and T. Strohmer, "General deviants: an analysis of perturbations in compressed sensing," IEEE Journal on Selected Topics in Signal Processing, vol. 4, no. 2, pp. 342-349, 2010.

[27] Y. Chi, L. L. Scharf, A. Pezeshki, and A. R. Calderbank, "Sensitivity to basis mismatch in compressed sensing," IEEE Transactions on Signal Processing, vol. 59, no. 5, pp. 2182-2195, 2011.

[28] O. Teke, A. C. Gurbuz, and O. Arikan, "A new OMP techniques for sparse recovery," in Proceedings of the 20th Signal Processing and Communications Applications Conference (SIU '12), Fethiye, Turkey, April 2012.
[29] A. S. Khwaja and X.-P. Zhang, "Compressed sensing based image formation of SAR/ISAR data in presence of basis mismatch," in Proceedings of the 2012 IEEE International Conference on Image Processing (ICIP '12), Orlando, Fla, USA, 2012.

[30] S. Yu, A. Shaharyar Khwaja, and J. Ma, "Compressed sensing of complex-valued data," Signal Processing, vol. 92, no. 2, pp. 357$362,2012$.

[31] G. Franceschetti and R. Lanari, Synthetic Aperture Radar Processing, CRC Press, Oxford, UK, 1999.

[32] E. G. Larsson and Y. Selén, "Linear regression with a sparse parameter vector," IEEE Transactions on Signal Processing, vol. 55, no. 2, pp. 451-460, 2007.

[33] M. Soumekh, Synthetic Aperture Radar Signal Processing, John Wiley and Sons, 1999.

[34] P. Tichavsky, C. H. Muravchik, and A. Nehorai, "Posterior Cramér-Rao bounds for discrete-time nonlinear filtering," IEEE Transactions on Signal Processing, vol. 46, no. 5, pp. 1386-1396, 1998.

[35] H. Zayyani, M. Babaie-Zadeh, and C. Jutten, "Bayesian cramerrao bound for noisynon-blind and blind compressed sensing," http://arxiv.org/abs/1005.4316. 

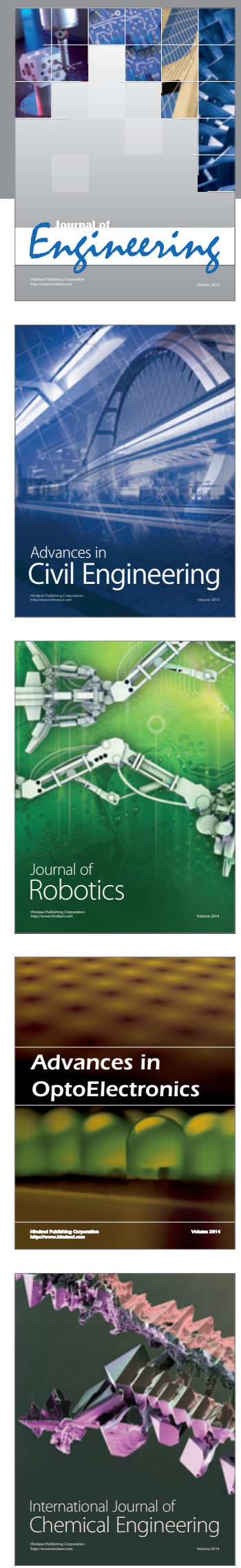

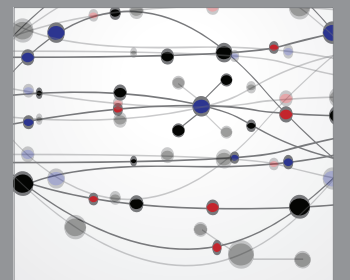

The Scientific World Journal
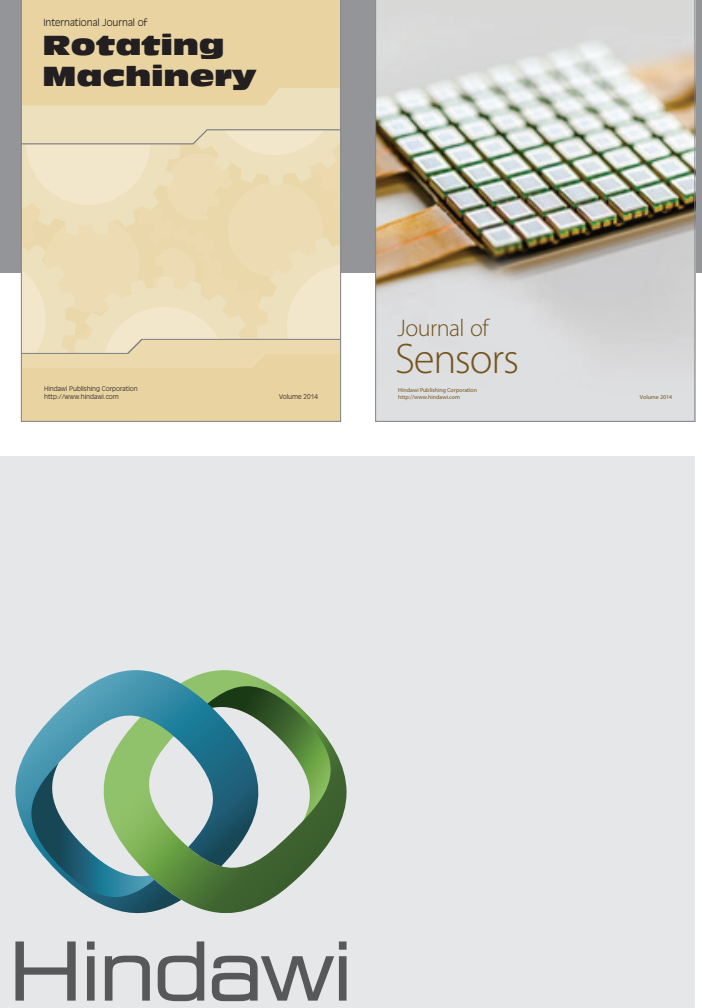

Submit your manuscripts at http://www.hindawi.com
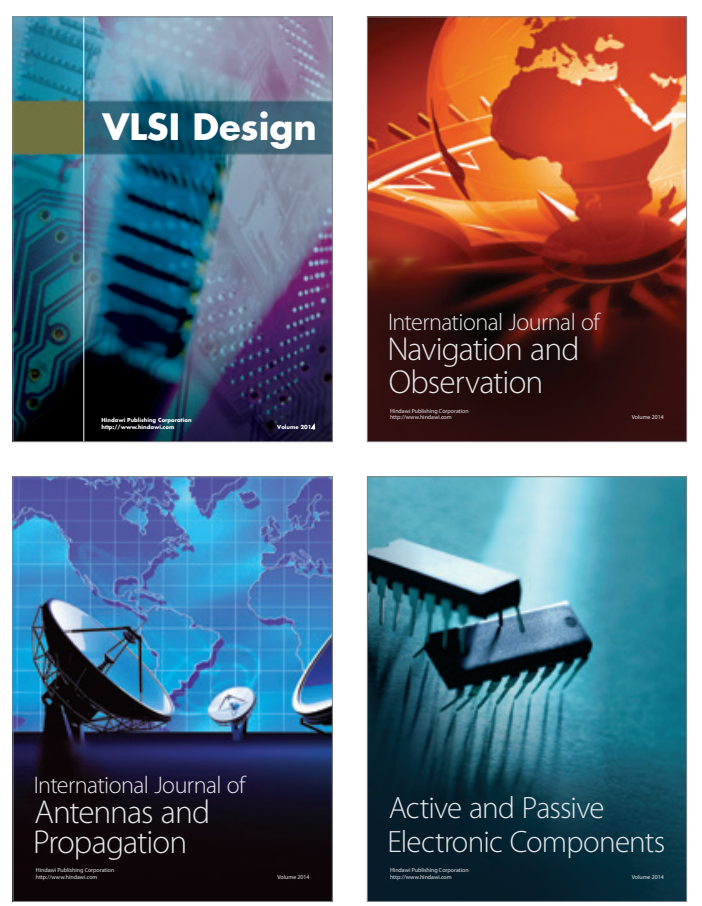
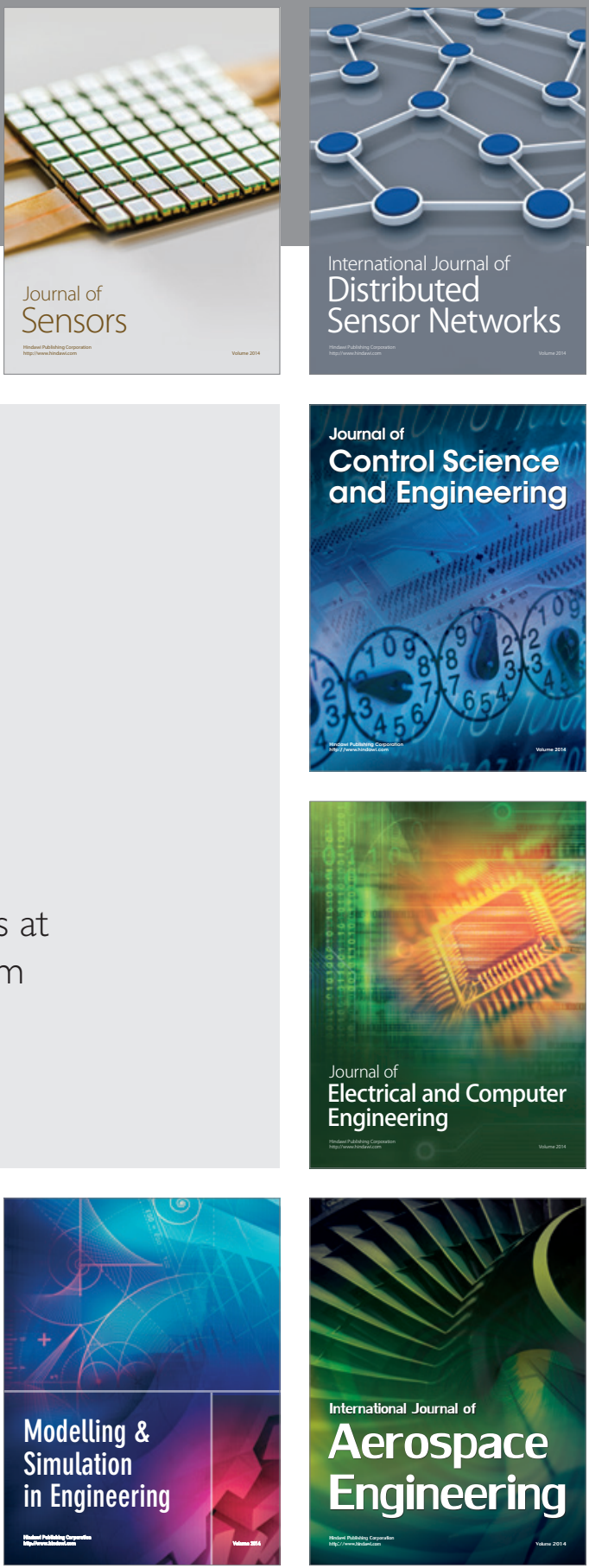

Journal of

Control Science

and Engineering
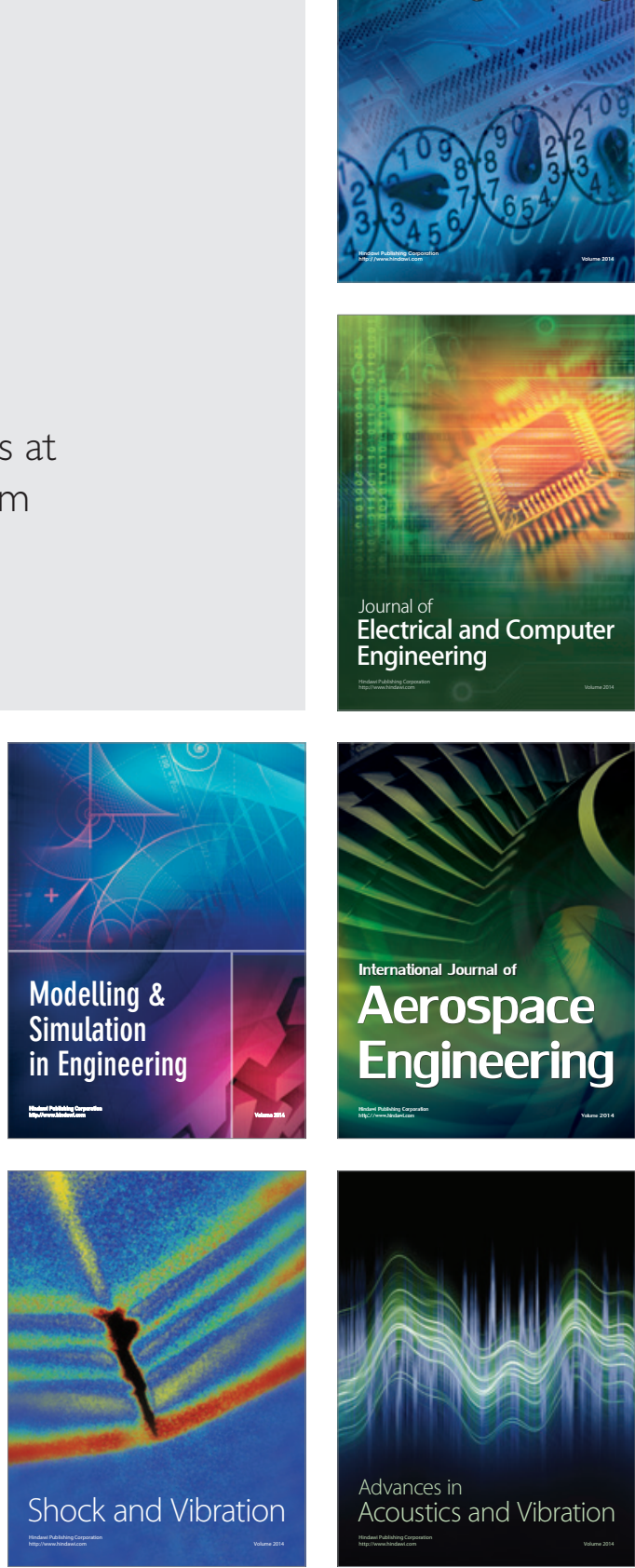